Sample Proficiency Test exercise

Armando Alcaraz, Hugh Gregg, Carolyn Koester

February 10, 2006

\title{
UCRL-TR-218830
}


This document was prepared as an account of work sponsored by an agency of the United States Government. Neither the United States Government nor the University of California nor any of their employees, makes any warranty, express or implied, or assumes any legal liability or responsibility for the accuracy, completeness, or usefulness of any information, apparatus, product, or process disclosed, or represents that its use would not infringe privately owned rights. Reference herein to any specific commercial product, process, or service by trade name, trademark, manufacturer, or otherwise, does not necessarily constitute or imply its endorsement, recommendation, or favoring by the United States Government or the University of California. The views and opinions of authors expressed herein do not necessarily state or reflect those of the United States Government or the University of California, and shall not be used for advertising or product endorsement purposes.

This work was performed under the auspices of the U.S. Department of Energy by University of California, Lawrence Livermore National Laboratory under Contract W-7405-Eng-48. 


\section{Sample Proficiency Test Exercise}

The current format of the OPCW proficiency tests has multiple sets of 2 samples sent to an analysis laboratory. In each sample set, one is identified as a sample, the other as a blank. This method of conducting proficiency tests differs from how an OPCW designated laboratory would receive authentic samples (a set of three containers, each not identified, consisting of the authentic sample, a control sample, and a blank sample).

This exercise was designed to test the reporting if the proficiency tests were to be conducted. As such, this is not an official OPCW proficiency test, and the attached report is one method by which LLNL might report their analyses under a more realistic testing scheme. Therefore, the title on the report "Report of the Umpteenth Official OPCW Proficiency Test” is meaningless, and provides a bit of whimsy for the analyses and readers of the report.

H. Gregg

A. Alcaraz

C. Koester 


\title{
ORGANISATION FOR THE PROHIBITION OF CHEMICAL WEAPONS
}

\author{
Report of the \\ Umpteenth Official OPCW \\ Proficiency Test
}

Laboratory code:

Total number of pages: $\quad 44^{1}$

${ }^{1}$ Total number of pages including cover page and all attachments 


\section{TABLE OF CONTENTS}

Cover page

Page no.

Table of contents

Summary

Participating Laboratory

Quality Assurance/Quality Control (QA/QC) ................................

Names and structures of all reported compounds ............................. 7

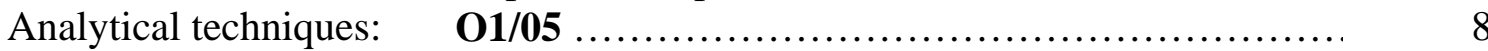

O2/05 ........................................ 9

Sample preparation description/flowchart ................................ 10

\section{Organic Sample 01}

Compound number $\mathbf{1}$

Analytical technique 1: GC/MS-EI ................................. 12

Analytical technique 2: GC/MS-CI .................................. 15

Analytical technique 3: GC/dFPD .................................. 18

Compound number 2

Analytical technique 1: GC/MS-EI .................................... 20

Analytical technique 2: GC/MS-CI .................................... 23

Analytical technique 3: GC/dFPD ................................... 26

\section{Organic Sample O2}

Compound number 3

Analytical technique 1: GC/MS-EI

Analytical technique 2: GC/MS-CI

Analytical technique 3: GC/dFPD

Compound number $\mathbf{4}$

Analytical technique 1: GC/MS-EI .................................. 36

Analytical technique 2: GC/MS-CI .................................. 39

Analytical technique 3: GC/ dFPD ................................... 42

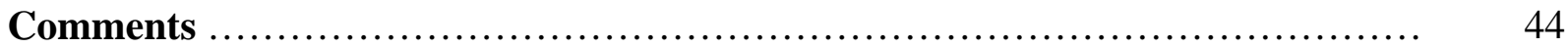




\section{SUMMARY: PARTICIPATING LABORATORY}

1. Participating laboratory

\begin{tabular}{|l|l|}
\hline Laboratory code & 05 \\
\hline $\begin{array}{l}\text { Name of the } \\
\text { laboratory/institute } \\
\text { participating in the test }\end{array}$ & Lawrence Livermore National Laboratory \\
\hline Contact person & Mr. Armando Alcaraz \\
\hline Address & $\begin{array}{l}\text { PO Box 808, M/S L-178 } \\
\text { 7000 East Avenue } \\
\text { Livermore, CA 94551 }\end{array}$ \\
\hline Telephone number & 925-423-6889 \\
\hline Fax number & 925-423-9014 \\
\hline Email address & Alcaraz1@llnl.gov \\
\hline
\end{tabular}

\section{Analysts and authentication}

\begin{tabular}{|c|c|c|c|c|c|}
\hline & Name & Title & Pages* & Date** & Signature** \\
\hline 1 & $\begin{array}{l}\text { Mr. Armando } \\
\text { Alcaraz }\end{array}$ & $\begin{array}{l}\text { Principal } \\
\text { Investigator }\end{array}$ & All & Feb. 3, 2006 & \\
\hline 2 & Dr. Hugh Gregg & $\begin{array}{l}\text { Co-PI, } \\
\text { Senior Chemist }\end{array}$ & All & Feb. 3, 2006 & \\
\hline 3 & Dr. Carolyn Koester & Research Scientist & $\begin{array}{l}\text { Prep \& } \\
\text { GC/MS }\end{array}$ & Feb. 3, 2006 & \\
\hline 4 & Dr. Phil Pagoria & Research Scientist & Synthesis & Feb. 3, 2006 & \\
\hline 5 & Dr. Robert Maxwell & Research Scientist & $\begin{array}{l}\text { NMR } \\
\text { analysis }\end{array}$ & Feb. 3, 2006 & \\
\hline 6 & $\begin{array}{l}\text { Ms. Tuijauna } \\
\text { Mitchell-Hall }\end{array}$ & QA Manager & $\mathrm{QA} / \mathrm{QC}$ & Feb. 3, 2006 & \\
\hline 7 & & & & & 0 \\
\hline 8 & & & & & \\
\hline 9 & & & & & \\
\hline
\end{tabular}

* Page numbers defining the responsibility area of the analyst;

** Date and signature of the responsible analyst; 


\section{SUMMARY: QUALITY ASSURANCE / QUALITY CONTROL (QA/QC)}

\section{Status of the laboratory (tick where applicable)}

$\bigotimes$ Accreditation accepted:

Year 2001 Accreditation body: American Association for Laboratory Accreditation

Scope of accreditation: $\underline{\text { Chemical }}$

Accreditation planned/pending:

Target year ___ Accreditation body:

Scope of accreditation:

Not accredited.

\section{Quality system (tick where applicable)}

$\bigotimes$ Described in a Quality Assurance Manual/Handbook. Quality system in accordance with:

$\square$ ISO $900 \_, \quad \square$ EN 4500__, $\$ ISO Guide 17025, $\square$ Other:

$\square$ No quality system. Please, fill in question number 3.

3. QA/QC Summary (Summary of the applied quality assurance and quality control (QA/QC) procedures concerning sample preparation, calibration, and analysis. Requested only from laboratories without a quality system). 


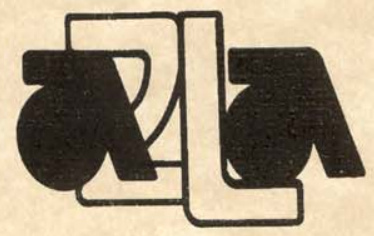

THE AMERICAN

ASSOCIATION

FOR LABORATORY

ACCREDITATION

\section{ACCREDITED LABORATORY}

\section{A2LA has accredited}

\section{LAWRENCE LIVERMORE NATIONAL}

LABORATORY

Livermore, CA

for technical competence in the field of

\section{Chemical Testing}

The accreditation covers the specific tests and types of tests listed on the agreed scope of accreditation. This laboratory meets the requirements of ISO/IEC 17025 1999 "General Requirements for the Competence of Testing and Calibration Laboratories" and any additional program requirements in the identified field of testing.

Presented this $12^{\text {th }}$ day of April 2004.
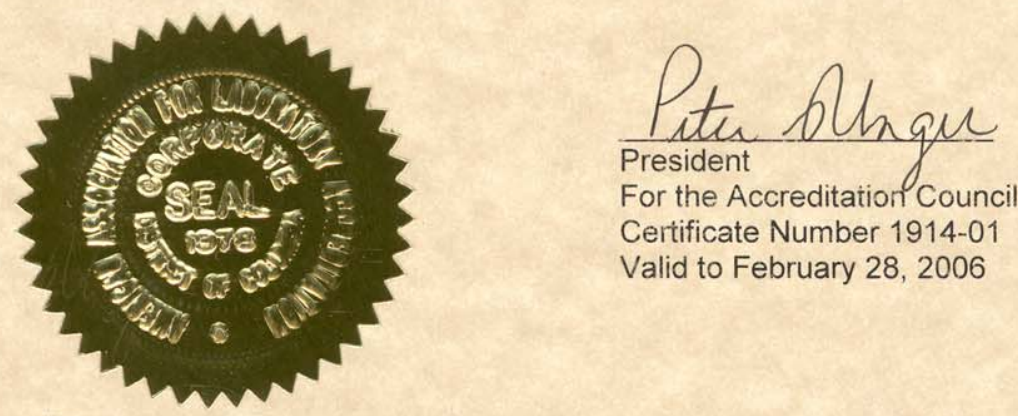

For the Accreditation Council

Certificate Number 1914-01

Valid to February 28, 2006

For tests or types of tests to which this accreditation applies, please refer to the laboratory's Chemical Scope of Accreditation. 


\title{
American Association for Laboratory Accreditation
}

\author{
SCOPE OF ACCREDITATION TO ISO/IEC 17025-1999 \\ LAWRENCE LIVERMORE NATIONAL LABORATORY \\ FORENSIC SCIENCE CENTER - OPCW PROJECT \\ 7000 East Avenue Mailstop L-178 \\ Livermore, CA 94550 \\ Armando Alcaraz Phone: 9254236889
}

\section{CHEMICAL}

Valid To: February 28, 2006

Certificate Number: $1914-01$

In recognition of the successful completion of the A2LA evaluation process, accreditation is granted to this laboratory to perform the following types of Qualitative Tests for Chemicals related to Chemical Warfare Convention (CWC) in unknown samples:

Sample Preparation

$\underline{\text { Test }}$

Spectroscopy

Nuclear magnetic resonance

Capillary zone electrophoresis / UV detection

Gas chromatography / Fourier Transform

Infrared Spectrometry

Chromatography

Gas chromatography / Element Specific Detectors

Gas chromatography / Mass Spectrometry

Liquid chromatography / Atmospheric pressure

Chemical Ionization / Mass Spectrometry

Liquid Chromatography / Electrospray Ionization Mass Spectrometry

Chain of Custody for Laboratory

Work Instructions for the Preparation of Test Samples for OPCW Proficiency Tests

(A2LA Cert. No. 1914-01) Revised 04/15/2004
BB-SP5, BB-SP6, BB-SP8, BB-SP9,

LL-SP1, LL-SP2, LL-SP3, LL-SP4

Procedures

BB-NMR 1

LL-CE1

BB-IR1

BB-GC1

BB-MS1

BB-MS4

LL-MS1

LL-CC1

QDOC/LAB/WI/ PT2

(Minus Section 11 Confirmation)

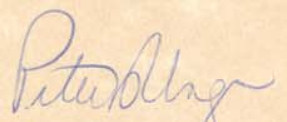

Page 1 of 1

5301 Buckeystown Pike, Suite 350 • Frederick, MD 21704-8373 • Phone: 301-644 3248 • Fax: 301-662 2974 
SUMMARY: NAMES AND STRUCTURES OF ALL REPORTED CHEMICALS

\begin{tabular}{|c|c|c|c|c|c|c|c|}
\hline Labo & atory co & 05 & & & & & \\
\hline $\begin{array}{l}\text { Sam. } \\
\text { code }\end{array}$ & $\begin{array}{c}\text { Chem. } \\
\text { no* }\end{array}$ & Chemical name & $\begin{array}{c}\text { Chemical } \\
\text { Abstract } \\
\text { number }\end{array}$ & Chemical Structure & $\begin{array}{l}\text { Molecular } \\
\text { formula }\end{array}$ & $\begin{array}{c}\text { Schedule } \\
\text { number }\end{array}$ & Comments $^{* *}$ \\
\hline $\mathrm{O} 1$ & 1 & Diethyl methylphosphonate & 683-08-9 & - & $\mathrm{C}_{5} \mathrm{H}_{13} \mathrm{O}_{3} \mathrm{P}$ & 2.B.04 & \\
\hline $\mathrm{O} 1$ & 2 & Dicyclohexyl methylphosphonate & 7040-53-1 & & $\mathrm{C}_{13} \mathrm{H}_{25} \mathrm{O}_{3} \mathrm{P}$ & 2.B.04 & \\
\hline $\mathrm{O} 2$ & 3 & Diisopropyl methylphosphonate & 1445-75-6 & & $\mathrm{C}_{7} \mathrm{H}_{17} \mathrm{O}_{3} \mathbf{P}$ & 2.B.04 & \\
\hline $\mathrm{O} 2$ & 4 & Diisopropyl ethylphosphonate & 1067-69-2 & & $\mathrm{C}_{8} \mathrm{H}_{19} \mathrm{O}_{3} \mathrm{P}$ & 2.B.04 & \\
\hline $\mathrm{O} 3$ & & No scheduled compounds & & & & & \\
\hline
\end{tabular}

* $\quad$ Chemical number defined by the participating laboratory and used throughout the report for the reported chemical.

** Explanation for the reporting of non-scheduled compounds, details can be added in the comment section of the report.

Note: There must be an unbroken chain of evidence linking each reported chemical to the original sample from which an aliquot was prepared and analyzed for the identification of this chemical. 


\section{SUMMARY: ANALYTICAL TECHNIQUES}

\section{Laboratory code: $\underline{05}$ Sample code(s):}

\begin{tabular}{|c|c|c|c|c|c|}
\hline $\begin{array}{l}\text { Chemical } \\
\text { number* }\end{array}$ & $\begin{array}{l}\text { Chemical } \\
\text { name }\end{array}$ & $\begin{array}{l}\text { Chemical } \\
\text { analysed as }\end{array}$ & $\begin{array}{l}\text { Analytical } \\
\text { technique }\end{array}$ & $\begin{array}{l}\text { Method } \\
\text { page no. }\end{array}$ & Aliquot name \\
\hline 1 & \begin{tabular}{|l|} 
Diethyl methylphosphonate \\
\end{tabular} & $\begin{array}{l}\text { original chemical } \\
\text { methylated } \\
\square \text { silylated } \\
\square \text { other: }\end{array}$ & $\begin{array}{l}\text { GC/MS-EI } \\
\text { GC/MS-CI } \\
\text { GC/dFPD }\end{array}$ & $\begin{array}{l}12 \\
15 \\
18\end{array}$ & $\begin{array}{l}\text { CW-1-159-1-O1 } \\
\text { CW-1-159-1-O1 } \\
\text { CW-1-159-1-O1 }\end{array}$ \\
\hline \multirow[t]{3}{*}{2} & Dicyclohexyl methylphosphonate & $\begin{array}{l}\text { original chemical } \\
\square \text { methylated } \\
\square \text { silylated } \\
\square \text { other: }\end{array}$ & $\begin{array}{l}\text { GC/MS-EI } \\
\text { GC/MS-CI } \\
\text { GC/dFPD }\end{array}$ & $\begin{array}{l}20 \\
23 \\
26\end{array}$ & $\begin{array}{l}\text { CW-1-159-1-O1 } \\
\text { CW-1-159-1-O1 } \\
\text { CW-1-159-1-O1 }\end{array}$ \\
\hline & & $\begin{array}{l}\square \text { original chemical } \\
\square \text { methylated } \\
\square \text { silylated } \\
\square \text { other: }\end{array}$ & & & \\
\hline & & $\begin{array}{l}\square \text { original chemical } \\
\text { methylated } \\
\text { m silylated } \\
\text { other: } \\
\end{array}$ & & & \\
\hline
\end{tabular}

* Chemical number defined by the participating laboratory (see Summary: Names and Structures of All Reported Chemical); 


\section{SUMMARY: ANALYTICAL TECHNIQUES}

Laboratory code: $\underline{05}$ Sample code(s):

\begin{tabular}{|c|c|c|c|c|c|}
\hline $\begin{array}{l}\text { Chemical } \\
\text { number* }\end{array}$ & Chemical name & \begin{tabular}{|l|}
$\begin{array}{l}\text { Chemical } \\
\text { analysed as }\end{array}$ \\
\end{tabular} & $\begin{array}{l}\text { Analytical } \\
\text { technique }\end{array}$ & $\begin{array}{l}\text { Method } \\
\text { page no. }\end{array}$ & Aliquot name \\
\hline 3 & Diisopropyl methylphosphonate & \begin{tabular}{|l}
$\square$ original chemical \\
$\square$ methylated \\
$\square$ silylated \\
$\square$ other: \\
\end{tabular} & $\begin{array}{l}\text { GC/MS-EI } \\
\text { GC/MS-CI } \\
\text { GC/dFPD }\end{array}$ & $\begin{array}{l}28 \\
31 \\
34\end{array}$ & $\begin{array}{l}\text { CW-1-159-2-O2 } \\
\text { CW-1-159-2-O2 } \\
\text { CW-1-159-2-O2 }\end{array}$ \\
\hline \multirow[t]{3}{*}{4} & Diisopropyl ethylphosphonate & \begin{tabular}{|l}
$\square$ original chemical \\
$\square$ methylated \\
$\square$ silylated \\
$\square$ other: \\
\end{tabular} & $\begin{array}{l}\text { GC/MS-EI } \\
\text { GC/MS-CI } \\
\text { GC/dFPD }\end{array}$ & $\begin{array}{l}36 \\
39 \\
42\end{array}$ & $\begin{array}{l}\text { CW-1-159-2-O2 } \\
\text { CW-1-159-2-O2 } \\
\text { CW-1-159-2-O2 }\end{array}$ \\
\hline & & \begin{tabular}{|l|l}
$\square$ & original chemical \\
$\square$ & methylated \\
$\square$ & silylated \\
$\square$ & other: \\
\end{tabular} & & & \\
\hline & & 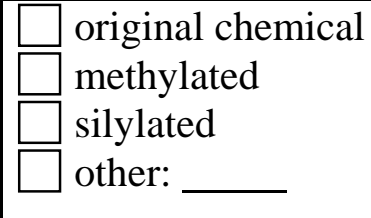 & & & \\
\hline
\end{tabular}

* Chemical number defined by the participating laboratory (see Summary: Names and Structures of All Reported Chemical); 


\section{SAMPLE PREPARATION DESCRIPTION}

Laboratory code: 05 Sample code(s): $\quad$ 01/05, 02/05, 03/05

\section{Sample preparation}

\begin{tabular}{|c|c|c|c|c|c|c|}
\hline $\begin{array}{l}\text { Sample/ } \\
\text { Aliquot Code }\end{array}$ & $\begin{array}{l}\text { Specification of Sample/ } \\
\text { Type of Sample Preparation }\end{array}$ & $\begin{array}{l}\text { Amount/ } \\
\text { Volume }\end{array}$ & Sample Preparation Procedures & \begin{tabular}{|l|} 
End \\
Volume \\
\end{tabular} & $\begin{array}{l}\text { Resulting Aliquot } \\
\text { Code }\end{array}$ & $\begin{array}{l}\text { Analytical } \\
\text { Technique(s) }\end{array}$ \\
\hline O1/05 & Organic & $0.2 \mathrm{~mL}$ & No sample workup. & $200 \mu \mathrm{L}$ & CW-1-159-1-O1 & $\begin{array}{l}\text { GC/MS-EI } \\
\text { GC/MS-CI } \\
\text { GC/dFPD }\end{array}$ \\
\hline $\mathrm{O} 2 / 05$ & None. & $0.2 \mathrm{~mL}$ & No sample workup. & $200 \mu \mathrm{L}$ & CW-1-159-2-O2 & $\begin{array}{l}\text { GC/MS-EI } \\
\text { GC/MS-CI } \\
\text { GC/dFPD }\end{array}$ \\
\hline$\overline{\mathrm{O} 3 / 05}$ & None. & $0.2 \mathrm{~mL}$ & No sample workup. & $200 \mu \mathrm{L}$ & CW-1-159-3-O3 & $\begin{array}{l}\text { GC/MS-EI } \\
\text { GC/MS-CI } \\
\text { GC/dFPD } \\
\end{array}$ \\
\hline & & & & & & \\
\hline & & & & & & \\
\hline & & & & & & \\
\hline & & & & & & \\
\hline & & & & & & \\
\hline
\end{tabular}

\section{Additional information}




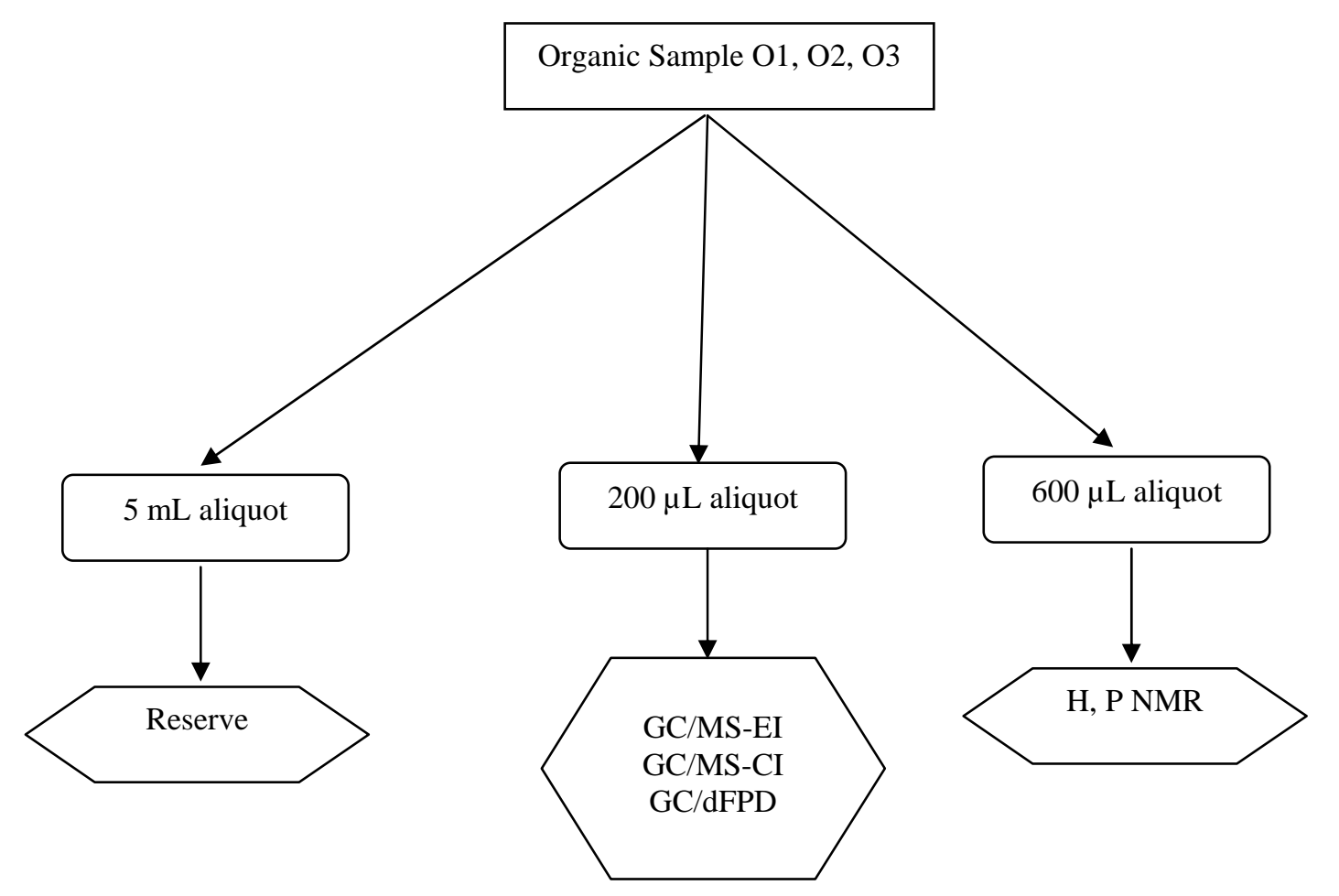

Note: This flowchart is for visualization only; see the preceding sample preparation description page for sample aliquot numbers 


\section{GC-EI-MS TECHNIQUE METHOD AND ANALYSIS DESCRIPTION}

Laboratory code: $\underline{05}$ Sample code(s): $\underline{\text { O1/05 }}$

Chemical number:

$\underline{1}$

Aliquot codes:

Sample: $\quad$ CW-1-159-1-O1

\begin{tabular}{|c|c|c|c|}
\hline $\begin{array}{l}\text { Instrument Manufacturer } \\
\text { and Type: }\end{array}$ & \multicolumn{3}{|c|}{ Agilent 6890/5973 GC/MSD } \\
\hline Carrier gas: & $\triangle \mathrm{He}$ & \multicolumn{2}{|l|}{$\square \mathrm{H}_{2} \quad \square$ Other: } \\
\hline Flow rate: & \multicolumn{3}{|c|}{$\mathrm{ml} / \mathrm{min}$} \\
\hline Flow control: & \multicolumn{3}{|c|}{$\square$ Constant Pressure $\quad \square$ Constant Flow } \\
\hline Injection mode: & \multicolumn{3}{|c|}{$\begin{array}{lll}\square \text { Split } & \rightarrow & \text { Split ratio }= \\
\square \text { Splitless } & \rightarrow \quad \text { Splitless time }=0.75 \mathrm{~min} .\end{array}$} \\
\hline Injector temperature: & \multicolumn{3}{|c|}{$250^{\circ} \mathrm{C}$} \\
\hline Column brand/phase: & \multicolumn{3}{|c|}{ Agilent HP-5MS: (5\%-Phenyl)-methylpolysiloxane } \\
\hline $\begin{array}{l}\text { Column Length } x \text { ID } x \\
\text { Film thickness: }\end{array}$ & \multicolumn{3}{|c|}{$30 \mathrm{~m} \times 0.25 \mathrm{~mm} \times 0.25 \mu \mathrm{m}$} \\
\hline $\begin{array}{l}\text { GC temperature } \\
\text { programme: }\end{array}$ & \multicolumn{3}{|c|}{$40^{\circ} \mathrm{C}(3 \mathrm{~min}), 8^{\circ} \mathrm{C} / \mathrm{min}, 300{ }^{\circ} \mathrm{C}(3 \mathrm{~min})$} \\
\hline Solvent delay time: & $3 \mathrm{~min}$ & Scan range: & $30-600 \mathrm{~m} / \mathrm{z}$ \\
\hline Electron energy: & $70 \mathrm{eV}$ & Scan time: & $0.7 \mathrm{~s}$ \\
\hline Ionisation polarity: & $\begin{array}{l}\square \text { Positive } \\
\square \text { Negative }\end{array}$ & Mass resolution: & $0.7 \mathrm{u}$ \\
\hline Comments: & & & \\
\hline
\end{tabular}

IDENTIFICATION

\begin{tabular}{|c|c|}
\hline Compound identified as: & $\begin{array}{l}\text { Q Original compound } \\
\square \text { Methyl ester derivative } \\
\square \text { TBDMS (t-Butyldimethylsilyl) derivative } \\
\square \text { TMS (Trimethylsilyl) derivative } \\
\square \text { Other derivative: }\end{array}$ \\
\hline $\begin{array}{l}\text { Retention parameter used for } \\
\text { (peak) identification: }\end{array}$ & $\begin{array}{l}\bigotimes \text { Retention time (Rt) } \\
\square \text { Scan number } \\
\end{array}$ \\
\hline \Compared to reference chemical: & Source : $\square$ Own Synthesis \\
\hline$\square$ Compared to library spectrum: & $\begin{array}{lll}\text { Source : } & \square \text { OCAD (code: } & \square \text { NIST } \\
& \square \text { Wiley } \quad \square \text { Own } \quad \square \text { Other: }\end{array}$ \\
\hline $\begin{array}{l}\square \text { Not compared to reference } \\
\text { chemical or library spectrum: }\end{array}$ & $\begin{array}{l}\text { Intense ions in spectrum are explained; interpretation is } \\
\text { supported by the spectral information derived from closely } \\
\text { related chemical(s): }\end{array}$ \\
\hline
\end{tabular}




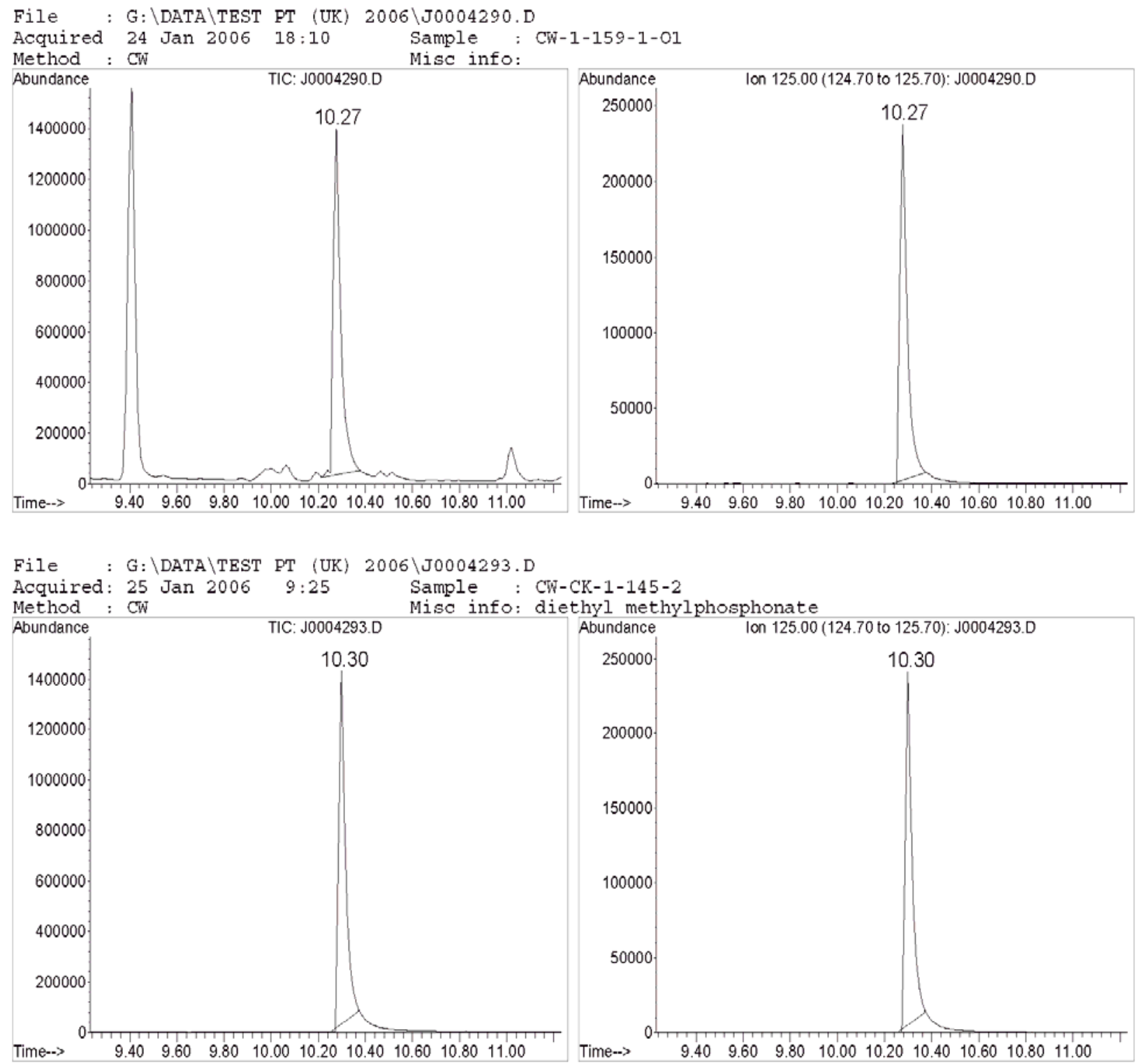

EI chromatograms supporting identification of compound 1; TIC on left; EIC (m/z 125) on right.

Top: Chromatograms of Organic sample, aliquot CW-1-159-1-O1 from O1/05, retention time 10.27 min.

Bottom: Chromatograms of authentic reference standard of Diethyl methylphosphonate, retention time 10.30 min. 
File $\quad$ D: \DATA \TEST PT (UK) 2006\J0004290.D

Acquired : 24 Jan 2006 18:10 using AcqMethod CW

Sample Name: CW-1-159-1-O1

Misc Info:

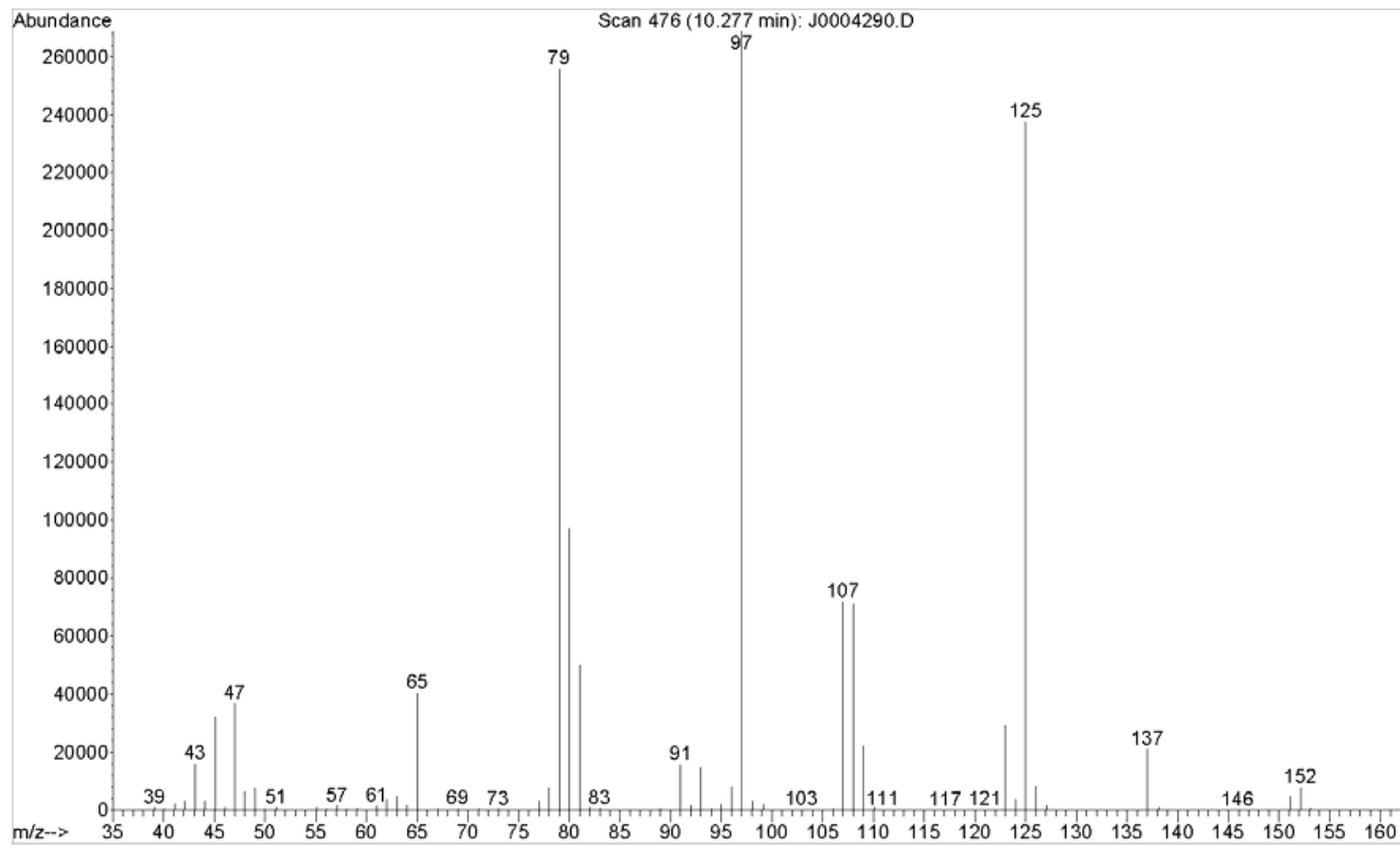

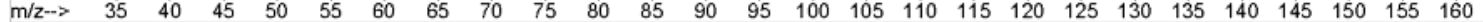

File :D: \DATA \TEST PT (UK) 2006\J0004293.D

Acquired : $25 \mathrm{Jan} 2006$ $9: 25$ using AcqMethod CW

Sample Name: CW-CK-1-145-2

Misc Info : diethyl methylphosphonate

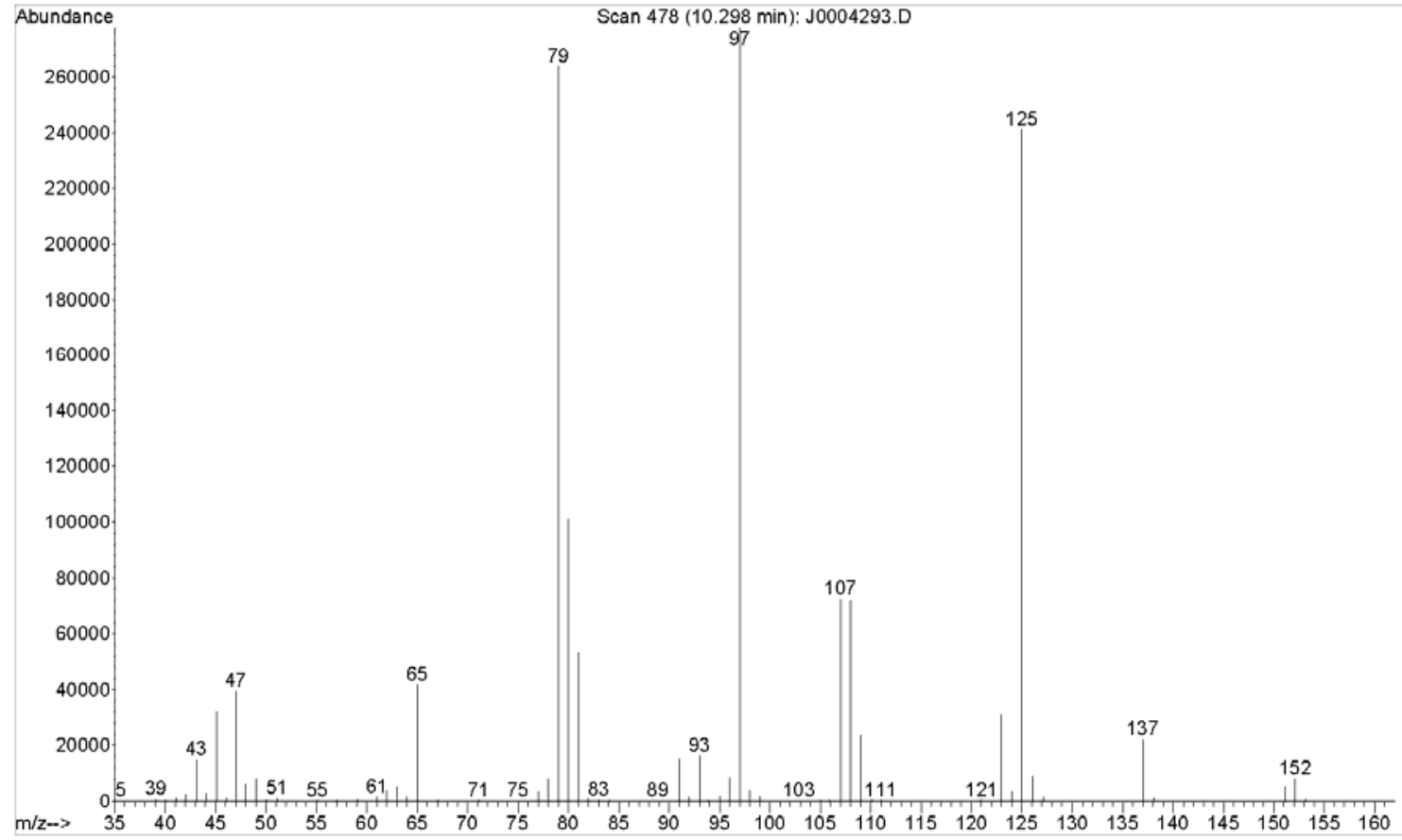

EI mass spectra of:

Top: $\quad$ Compound 1 in Organic sample 01/05, aliquot CW-1-159-1-01

Bottom: Authentic reference standard of Diethyl methylphosphonate corresponding to compound 1

(MW: 152) 


\section{GC-CI-MS TECHNIQUE METHOD AND ANALYSIS DESCRIPTION}

Laboratory code: $\underline{05}$ Sample code(s): $\underline{01 / 05}$

Chemical number: $\quad \underline{1}$

\begin{tabular}{|l|l|}
\hline \multicolumn{2}{|l|}{ Aliquot codes: } \\
\hline Sample: & $\mathrm{CW}-1-159-1-01$ \\
\hline
\end{tabular}

ANALYSIS METHOD

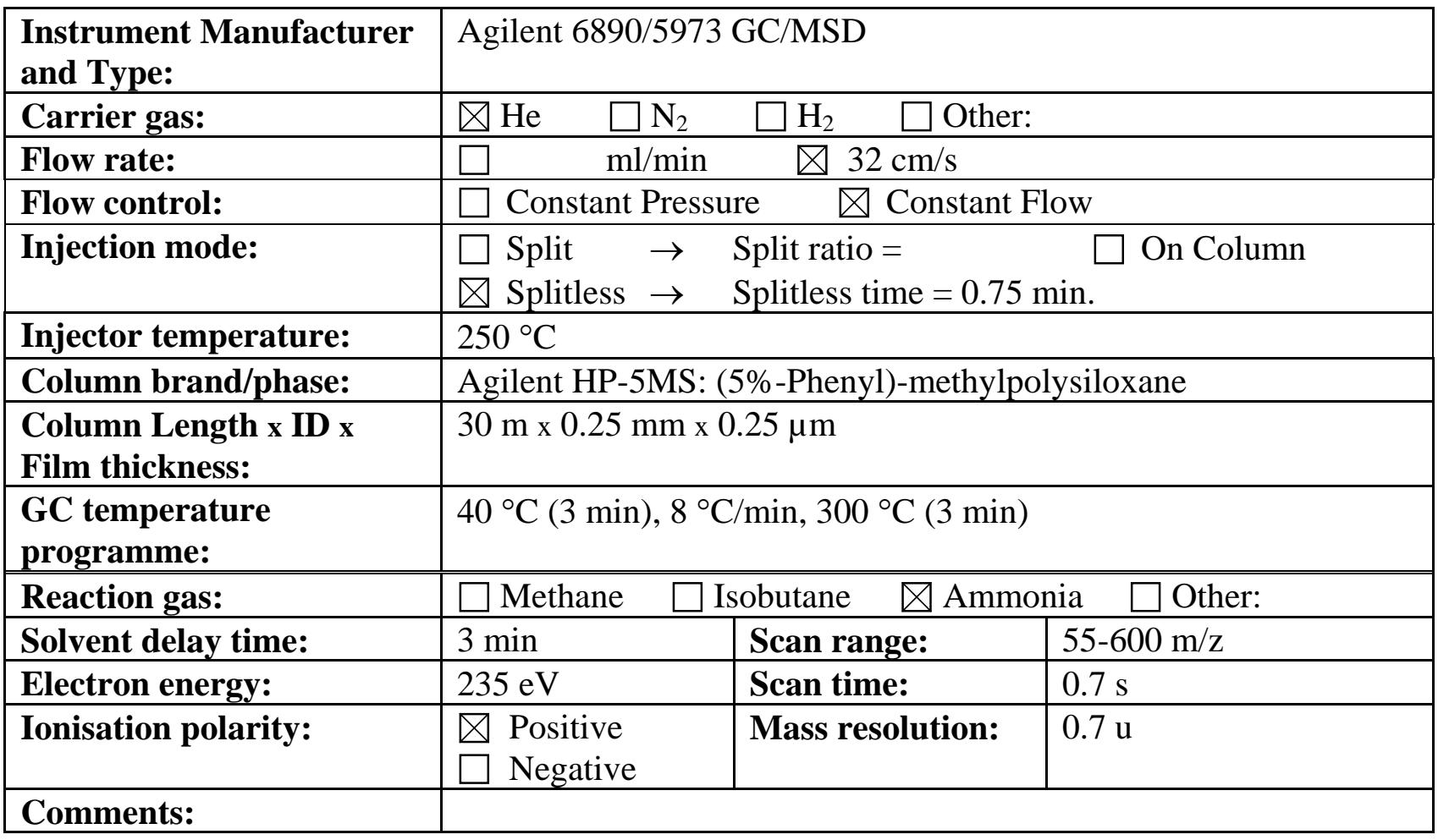

IDENTIFICATION

\begin{tabular}{|l|l|}
\hline Compound identified as: & $\square$ Original compound \\
& $\square$ Methyl ester derivative \\
& $\square$ TBDMS (t-Butyldimethylsilyl) derivative \\
& $\square$ TMS (Trimethylsilyl) derivative \\
& $\square$ Other derivative: \\
\hline $\begin{array}{l}\text { Retention parameter used for } \\
\text { (peak) identification: }\end{array}$ & $\square$ Retention time (Rt) \\
\hline \hline$\square$ Compared to reference chemical: & Source : $\square$ Own Synthesis $\square$ Commercial \\
\hline $\begin{array}{l}\text { Not compared to reference } \\
\text { chemical or library spectrum: }\end{array}$ & Intense ions in spectrum are explained \\
\hline Comments: & $\square$ RT GC/MS-EI \\
\hline
\end{tabular}




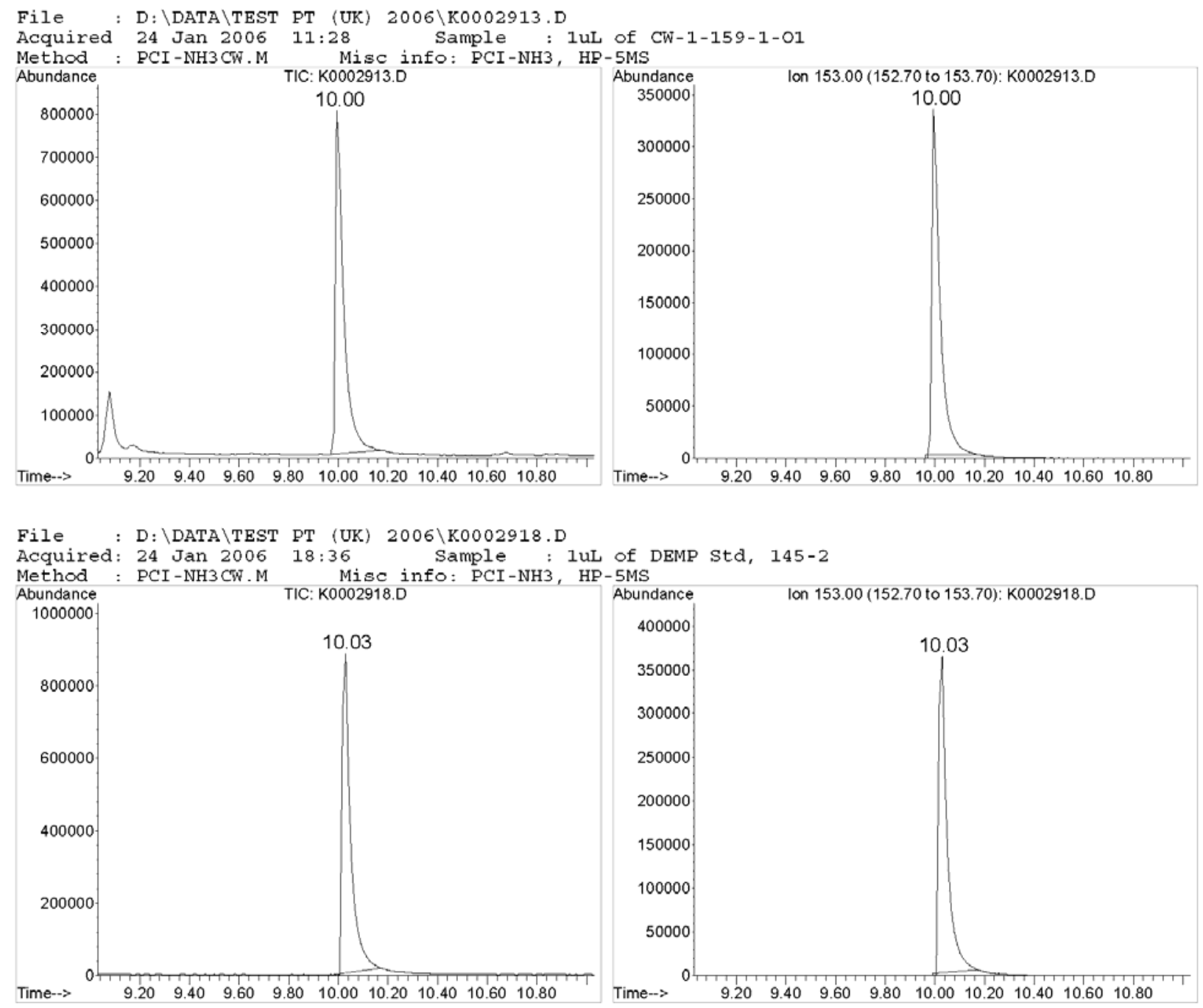

CI chromatograms supporting identification of compound 1; TIC on left; EIC (m/z 153) on right.

Top: $\quad$ Chromatograms of Organic sample, aliquot CW-1-159-1-O1 from 01/05, retention time 10.00 min.

Bottom: Chromatograms of authentic reference standard of Diethyl methylphosphonate, retention time 10.03 min. 
File $\quad$ :D: $\backslash$ DATA $\backslash$ TEST PT (UK) 2006 $\backslash$ K0002913.D

Acquired : 24 Jan $200611: 28$ Using AcqMethod PCI-NH3CW.M

Sample Name: 1uL of CW-1-159-1-01

Misc Info : PCI-NH3, HP-5MS

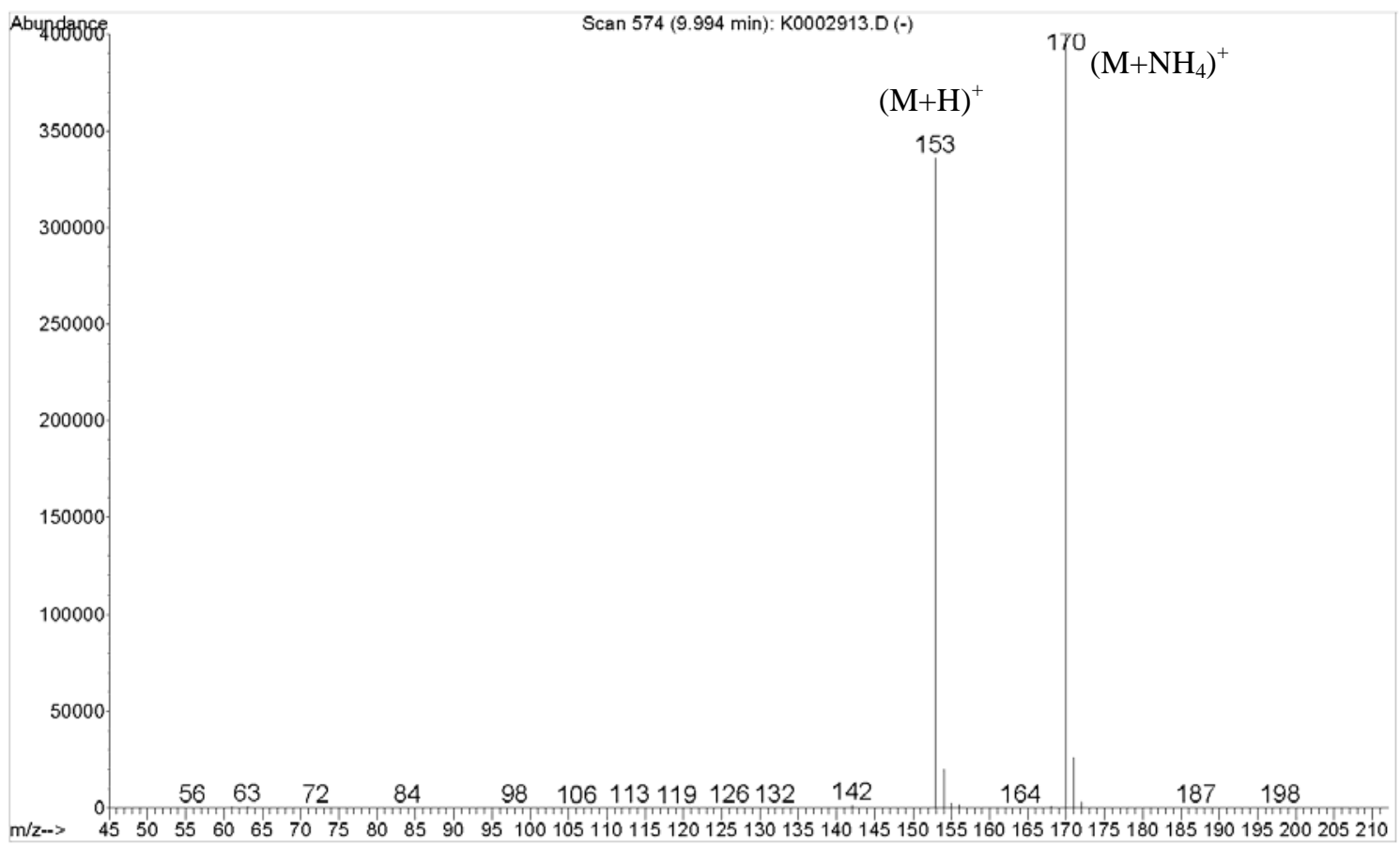

File $\quad$ :D: \DATA \TEST PT (UK) 2006\K0002918.D

Acquired : 24 Jan 2006 18:36 using AcqMethod PCI-NH3CW.M

Sample Name: 1uL of DEMP std, 145-2

Misc Info : PCI-NH3, HP-5MS

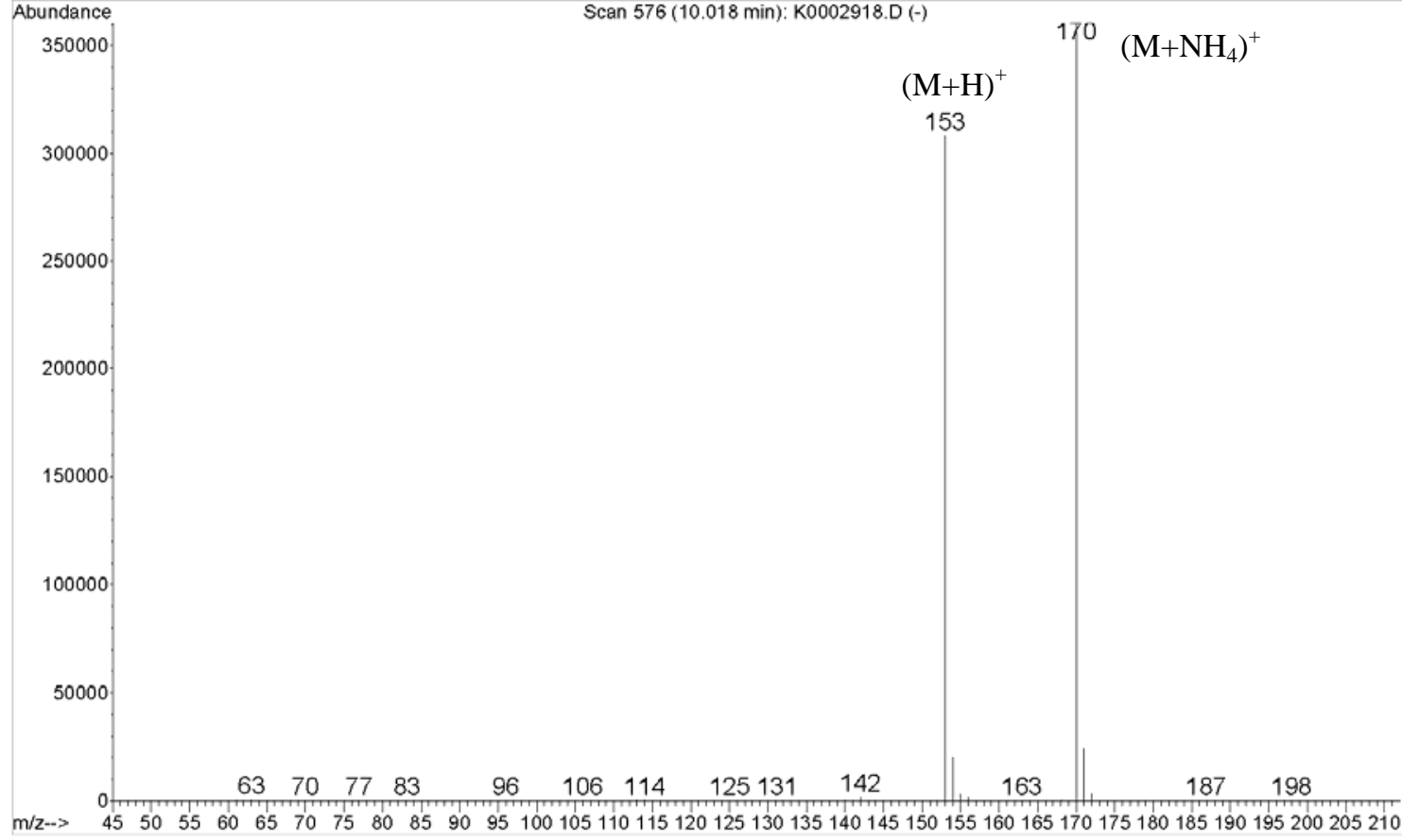

CI mass spectrum of:

Top: $\quad$ Compound 1 in Organic sample 01/05, aliquot CW-1-159-1-01

Bottom: Authentic reference standard of Diethyl methylphosphonate corresponding to compound 1

(MW: 152) 


\section{GAS CHROMATOGRAPHY TECHNIQUE METHOD AND ANALYSIS DESCRIPTION}

Laboratory code: $\underline{05}$ Sample code(s): $\underline{\text { 01/05 }}$ Chemical number: $\underline{1}$ Aliquot codes:

Sample: $\quad$ CW-1-159-1-01

ANALYSIS METHOD

\begin{tabular}{|c|c|}
\hline $\begin{array}{l}\text { Instrument Manufacturer } \\
\text { and Type: }\end{array}$ & Agilent 6890 GC dual FPD \\
\hline Carrier gas: & $\begin{array}{lll}\mathrm{He} & \square \mathrm{N}_{2} \quad \square \mathrm{H}_{2} \quad \square \text { Other: }\end{array}$ \\
\hline Flow rate: & $\bigotimes 32 \mathrm{~cm} / \mathrm{s}$ \\
\hline Flow control: & $\square$ Constant Pressure $\quad \bigotimes$ Constant Flow \\
\hline Injection mode: & $\begin{array}{lll}\square \text { Split } & \rightarrow & \text { Split ratio }= \\
\square \text { Splitless } & \rightarrow \quad \text { Splitless time }=0.75 \mathrm{~min} .\end{array}$ \\
\hline Injector temperature: & $250^{\circ} \mathrm{C}$ \\
\hline Column brand/phase: & Agilent HP-5MS: (5\%-Phenyl)-methylpolysiloxane \\
\hline $\begin{array}{l}\text { Column Length } \mathrm{x} \text { ID } \mathrm{x} \\
\text { Film thickness: }\end{array}$ & $30 \mathrm{~m} \times 0.25 \mathrm{~mm} \times 0.25 \mu \mathrm{m}$ \\
\hline $\begin{array}{l}\text { GC temperature } \\
\text { programme: }\end{array}$ & $40^{\circ} \mathrm{C}(3 \mathrm{~min}), 8^{\circ} \mathrm{C} / \mathrm{min}, 300^{\circ} \mathrm{C}(3 \mathrm{~min})$ \\
\hline Detector: & $\begin{array}{lll}\square \text { AED } & \rightarrow & \text { Element(s) }= \\
\square \text { NPD } & \\
\square \text { FPD } & \rightarrow & \square \text { P-mode } \\
\square \text { Other: } & & \end{array}$ \\
\hline
\end{tabular}

IDENTIFICATION

\begin{tabular}{|l|l|}
\hline Chemical identified as: & $\begin{array}{l}\text { O Original Chemical } \\
\square \text { Methyl ester derivative } \\
\square \text { TBDMS (t-Butyldimethylsilyl) derivative } \\
\square \text { TMS (Trimethylsilyl) derivative } \\
\square \text { Other derivative: }\end{array}$ \\
\hline $\begin{array}{l}\text { Retention parameter used } \\
\text { for (peak) identification: }\end{array}$ & $\square$ Retention time (Rt) $\square$ Scan number \\
\hline $\begin{array}{l}\text { QCompared to reference } \\
\text { chemical: }\end{array}$ & Source of Reference: $\square$ Own Synthesis $\quad$ Commercial \\
\hline $\begin{array}{l}\square \text { Compared to library } \\
\text { RI: }\end{array}$ & $\begin{array}{l}\text { Measured RI of identified Chemical }= \\
\text { RI OCAD }=\end{array}$ \\
\hline Comments & \\
\hline
\end{tabular}




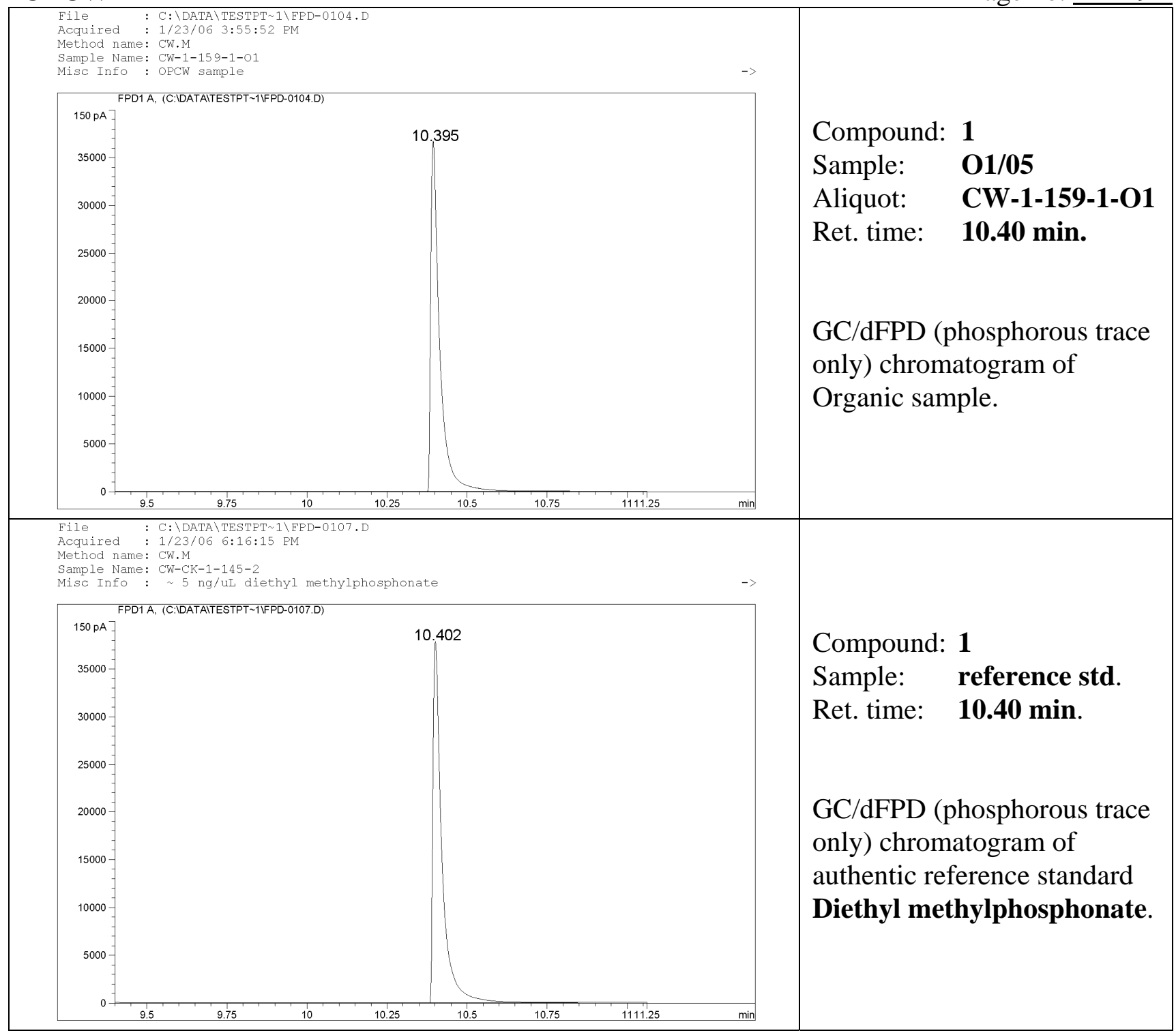




\section{GC-EI-MS TECHNIQUE METHOD AND ANALYSIS DESCRIPTION}

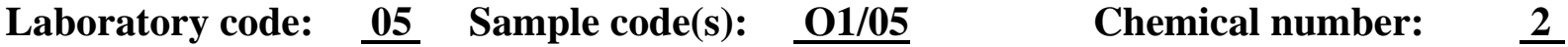

Aliquot codes:

Sample: $\quad$ CW-1-159-1-01

ANALYSIS METHOD

\begin{tabular}{|c|c|c|c|}
\hline $\begin{array}{l}\text { Instrument Manufacturer } \\
\text { and Type: }\end{array}$ & \multicolumn{3}{|c|}{ Agilent 6890/5973 GC/MSD } \\
\hline Carrier gas: & $\square \mathrm{N}_{2}$ & \multicolumn{2}{|l|}{$\square \mathrm{H}_{2} \quad \square$ Other: } \\
\hline Flow rate: & \multicolumn{3}{|c|}{ \32 cm/s } \\
\hline Flow control: & \multicolumn{3}{|c|}{$\square$ Constant Pressure $\quad \square$ Constant Flow } \\
\hline Injection mode: & $\begin{array}{ll}\square \text { Split } & \rightarrow \\
\bigotimes \text { Splitless } & \rightarrow\end{array}$ & \multicolumn{2}{|c|}{$\begin{array}{l}\text { Split ratio }=\quad \square \text { On Column } \\
\text { Splitless time = } 0.75 \mathrm{~min} .\end{array}$} \\
\hline Injector temperature: & \multicolumn{3}{|l|}{$250^{\circ} \mathrm{C}$} \\
\hline Column brand/phase: & \multicolumn{3}{|c|}{ Agilent HP-5MS: (5\%-Phenyl)-methylpolysiloxane } \\
\hline $\begin{array}{l}\text { Column Length } \mathrm{x} \text { ID } \mathrm{x} \\
\text { Film thickness: } \\
\end{array}$ & \multicolumn{3}{|c|}{$30 \mathrm{~m} \times 0.25 \mathrm{~mm} \times 0.25 \mu \mathrm{m}$} \\
\hline $\begin{array}{l}\text { GC temperature } \\
\text { programme: }\end{array}$ & \multicolumn{3}{|c|}{$40{ }^{\circ} \mathrm{C}(3 \mathrm{~min}), 8{ }^{\circ} \mathrm{C} / \mathrm{min}, 300{ }^{\circ} \mathrm{C}(3 \mathrm{~min})$} \\
\hline Solvent delay time: & $3 \mathrm{~min}$ & Scan range: & $30-600 \mathrm{~m} / \mathrm{z}$ \\
\hline Electron energy: & $70 \mathrm{eV}$ & Scan time: & $0.7 \mathrm{~s}$ \\
\hline Ionisation polarity: & $\begin{array}{l}\square \text { Positive } \\
\square \text { Negative }\end{array}$ & Mass resolution: & $0.7 \mathrm{u}$ \\
\hline
\end{tabular}

IDENTIFICATION

\begin{tabular}{|c|c|}
\hline Compound identified as: & $\begin{array}{l}\text { Q Original compound } \\
\square \text { Methyl ester derivative } \\
\square \text { TBDMS (t-Butyldimethylsilyl) derivative } \\
\square \text { TMS (Trimethylsilyl) derivative } \\
\square \text { Other derivative: }\end{array}$ \\
\hline $\begin{array}{l}\text { Retention parameter used for } \\
\text { (peak) identification: }\end{array}$ & $\begin{array}{l}\bigotimes \text { Retention time (Rt) } \\
\square \text { Scan number }\end{array}$ \\
\hline \ Compared to reference chemical: & Source : $\square$ Own Synthesis $\quad$ Commercial \\
\hline$\square$ Compared to library spectrum: & $\begin{array}{lll}\text { Source : } & \square \text { OCAD (code: } & \square \text { NIST } \\
& \square \text { Wiley } \quad \square \text { Own } & \square \text { Other: }\end{array}$ \\
\hline $\begin{array}{l}\square \text { Not compared to reference } \\
\text { chemical or library spectrum: }\end{array}$ & $\begin{array}{l}\text { Intense ions in spectrum are explained; interpretation is } \\
\text { supported by the spectral information derived from closely } \\
\text { related chemical(s): }\end{array}$ \\
\hline
\end{tabular}




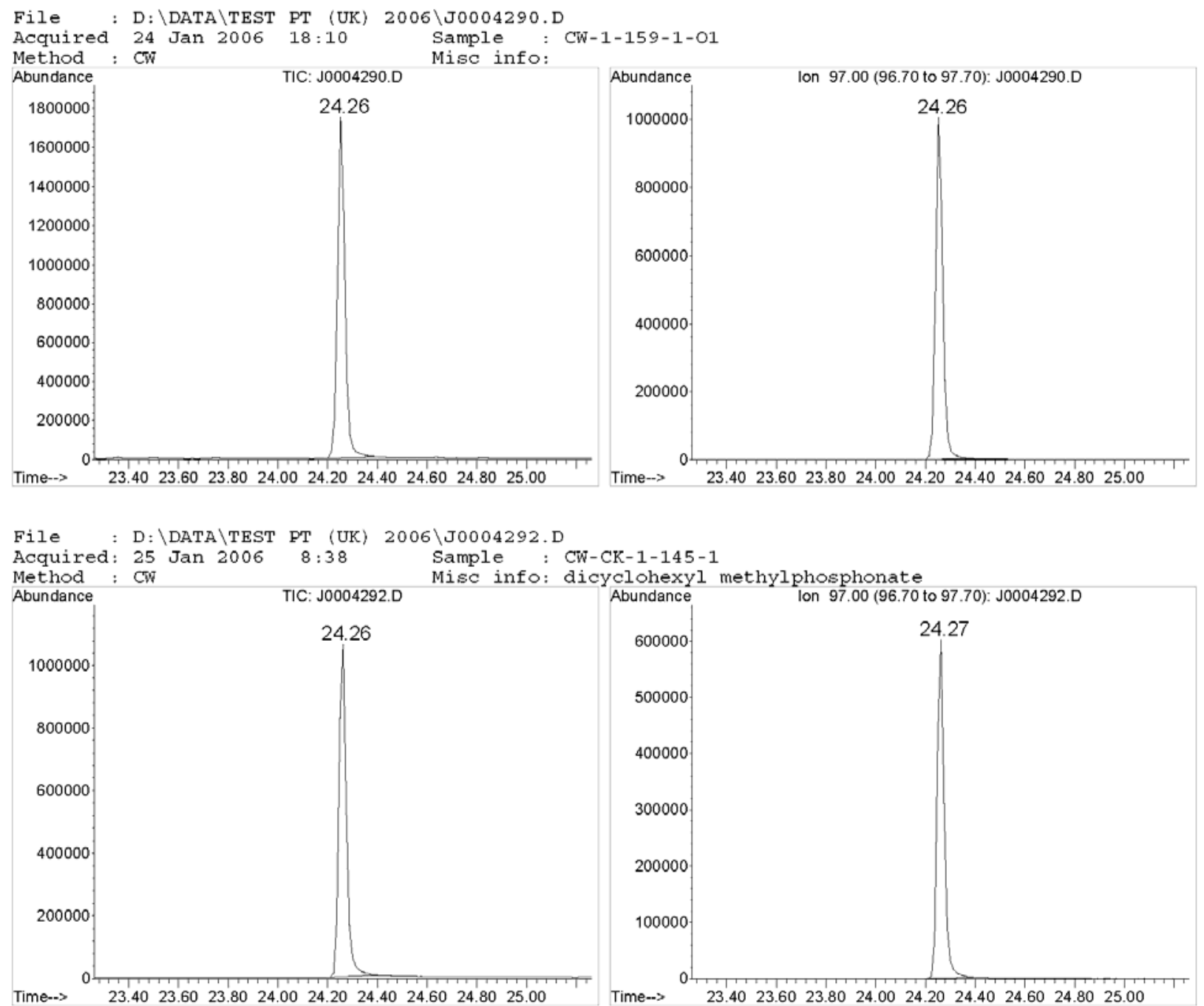

EI chromatograms supporting identification of compound 2; TIC on left; EIC (m/z 97) on right.

Top: $\quad$ Chromatograms of Organic sample, aliquot CW-1-159-1-O1 from 01/05, retention time 24.26 min.

Bottom: Chromatograms of authentic reference standard of Dicyclohexyl methylphosphonate, retention time 24.27 min. 
File :D: \DATA \TEST PT (UK) 2006 JJ0004290.D

Acquired : 24 Jan 2006 18:10 using AcqMethod CW

Sample Name: CW-1-159-1-O1

Misc Info :

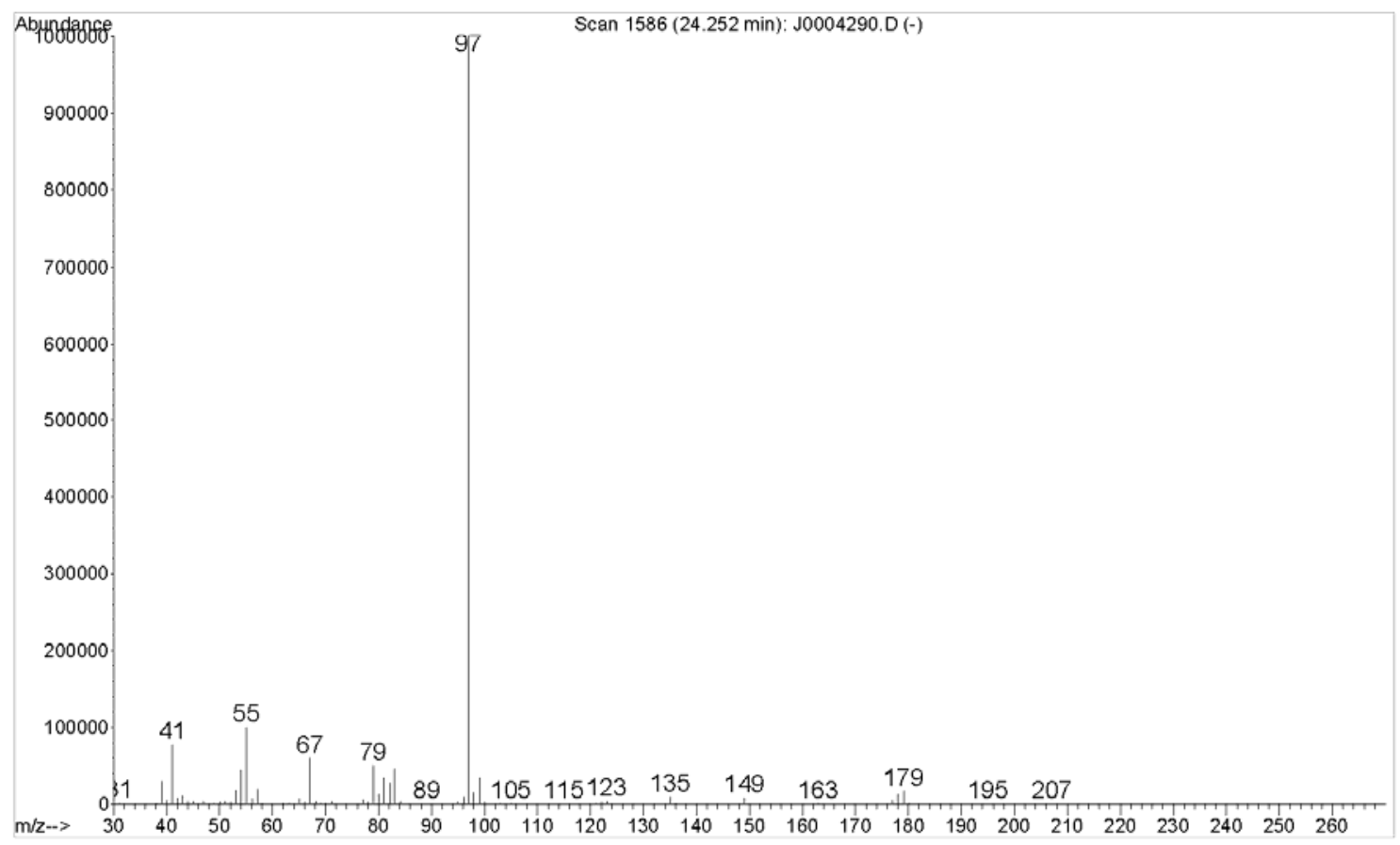

File :D: \DATA \TEST PT (UK) 2006\J0004292.D

Acquired : $25 \mathrm{Jan} 2006 \quad 8: 38$ using AcqMethod CW

Sample Name: CW-CK-1-145-1

Misc Info : dicyclohexyl methylphosphonate

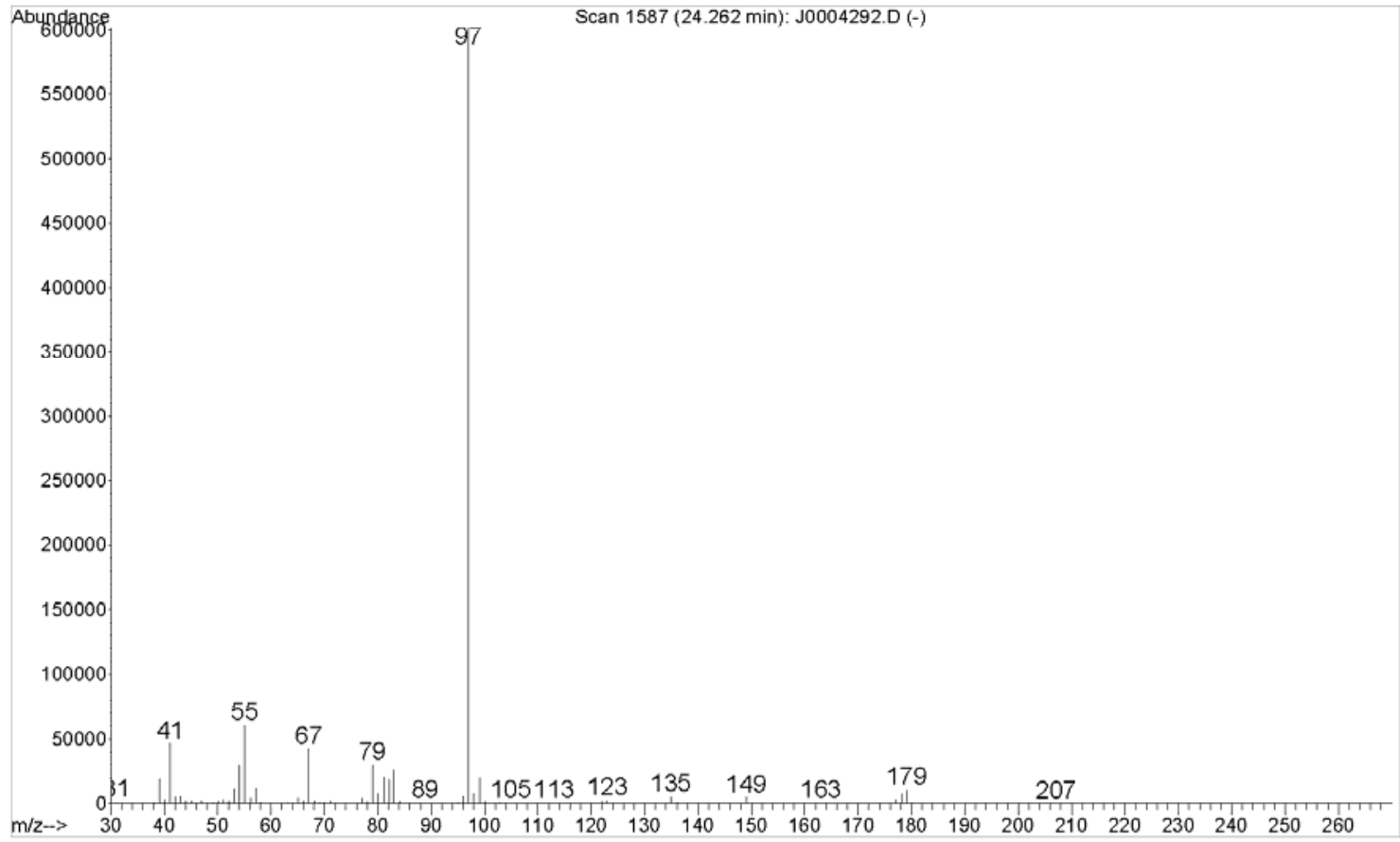

EI mass spectra of:

Top: $\quad$ Compound 2 in Organic sample 01/05, aliquot CW-1-159-1-O1

Bottom: Authentic reference standard of Dicyclohexyl methylphosphonate corresponding to compound 2 (MW: 260) 


\section{GC-CI-MS TECHNIQUE METHOD AND ANALYSIS DESCRIPTION}

Laboratory code: $\underline{05}$ Sample code(s): $\underline{\text { O1/05 }}$

Chemical number:

$\underline{2}$

Aliquot codes:

Sample: $\quad$ CW-1-159-1-O1

ANALYSIS METHOD

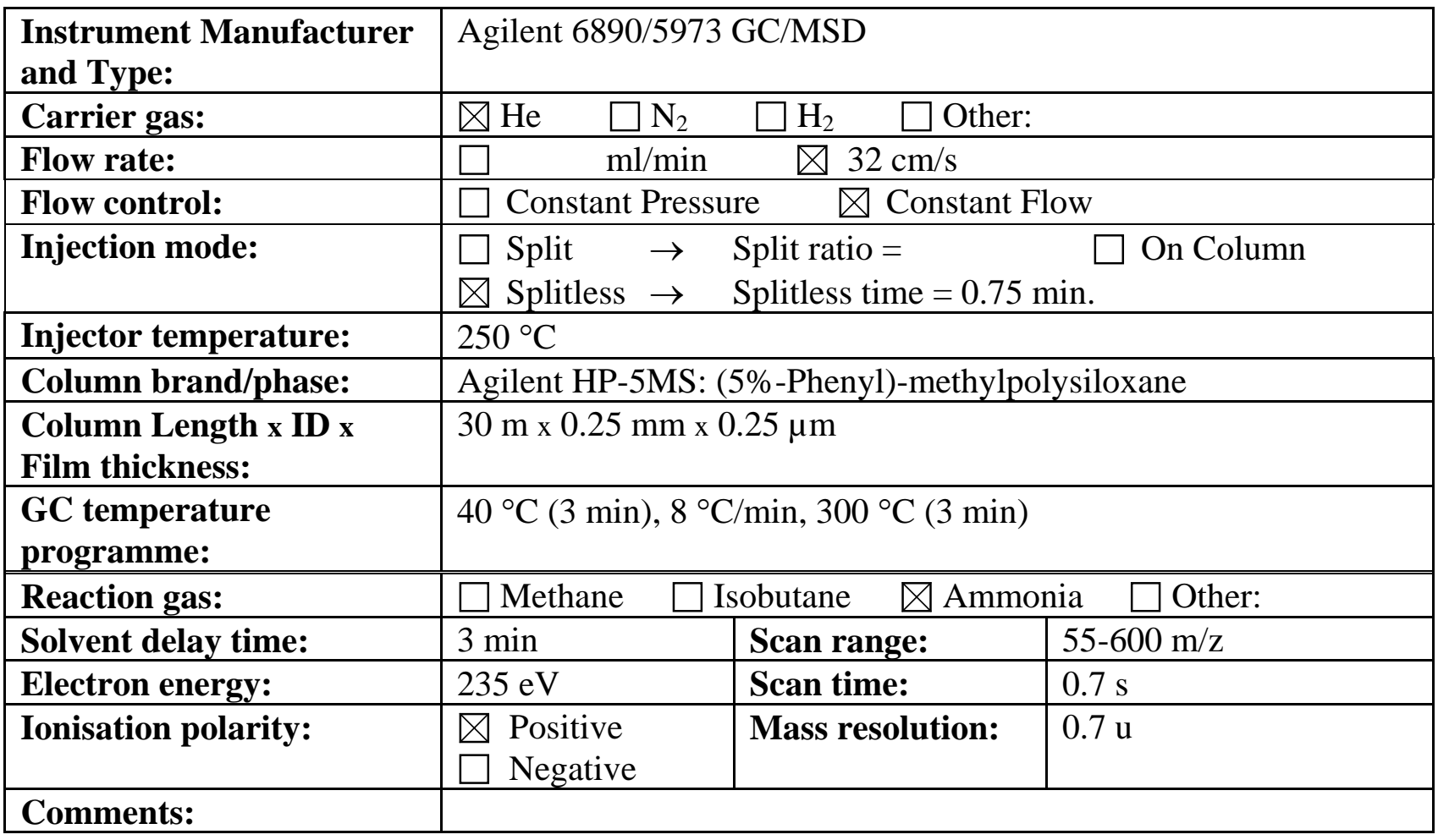

IDENTIFICATION

\begin{tabular}{|l|l|}
\hline Compound identified as: & Q Original compound \\
& $\square$ Methyl ester derivative \\
& $\square$ TBDMS (t-Butyldimethylsilyl) derivative \\
& $\square$ TMS (Trimethylsilyl) derivative \\
& $\square$ Other derivative: \\
\hline $\begin{array}{l}\text { Retention parameter used for } \\
\text { (peak) identification: }\end{array}$ & $\square$ Retention time (Rt) \\
\hline \hline$\square$ Compared to reference chemical: & Source : $\square$ Own Synthesis $\quad$ Commercial \\
\hline $\begin{array}{l}\text { Not compared to reference } \\
\text { chemical or library spectrum: }\end{array}$ & Intense ions in spectrum are explained \\
\hline Comments: & $\square$ RT GC/MS-EI \\
\hline
\end{tabular}




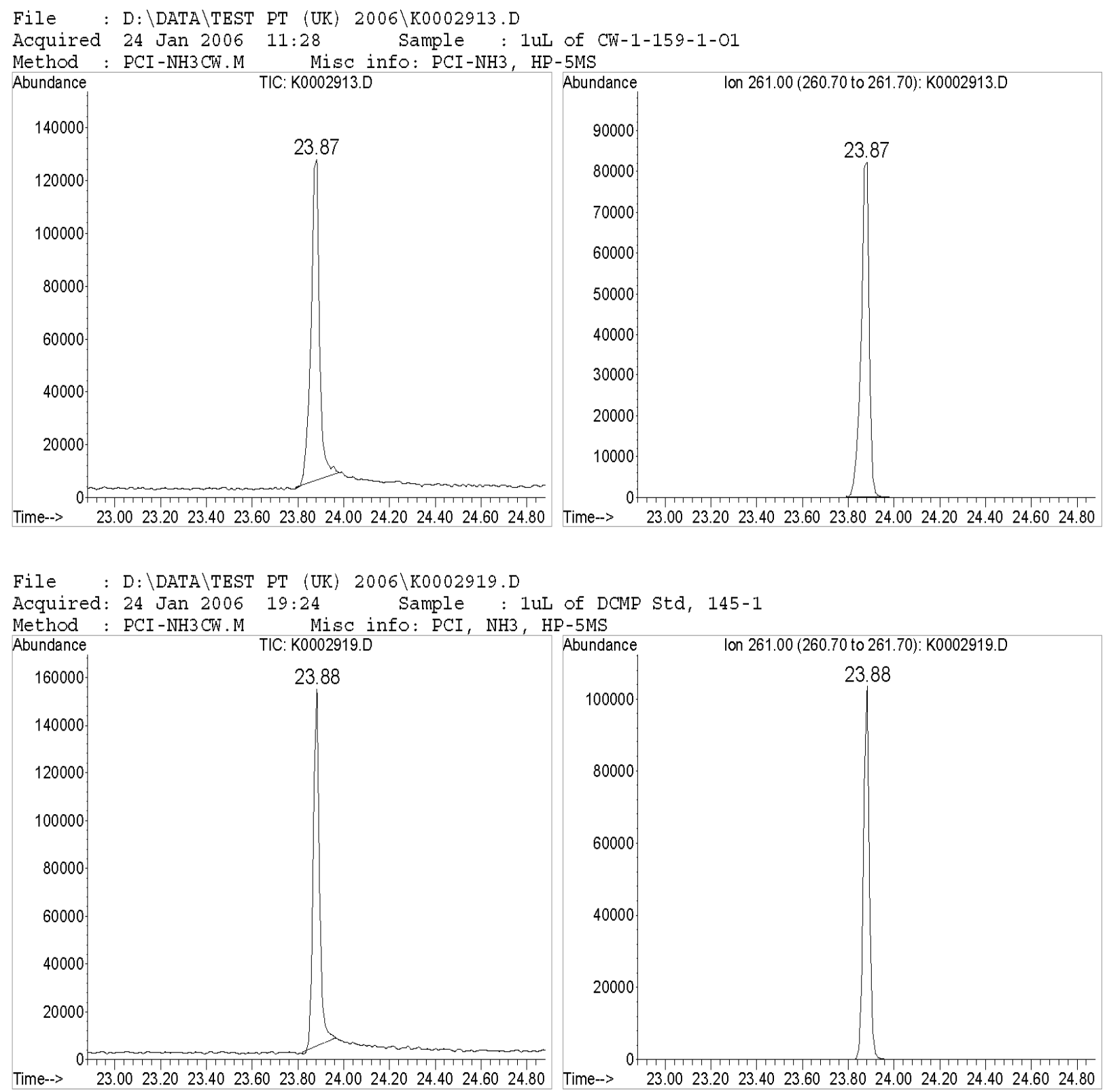

CI chromatograms supporting identification of compound 2; TIC on left; EIC (m/z 261) on right.

Top: $\quad$ Chromatograms of Organic sample, aliquot CW-1-159-1-O1 from 01/05, retention time 23.87 min.

Bottom: Chromatograms of authentic reference standard of Dicyclohexyl methylphosphonate, retention time $23.88 \mathrm{~min}$. 
File $\quad$ :D: \DATA \TEST PT (UK) 2006\K0002913.D

Acquired : 24 Jan 2006 11:28 using AcqMethod PCI -NH3CW.M

Sample Name: 1uL of CW-1-159-1-01

Misc Info : PCI-NH3, HP-5MS

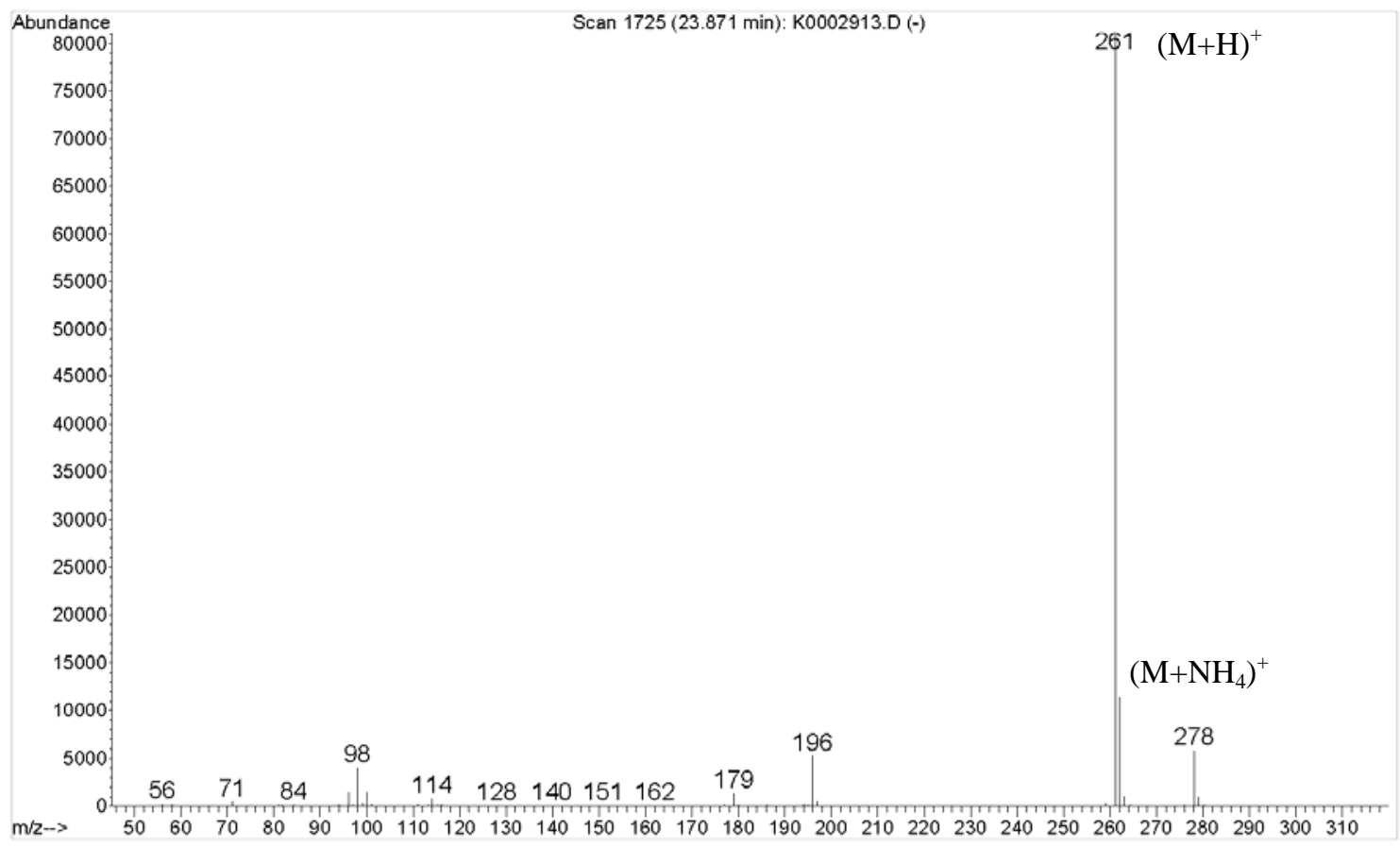

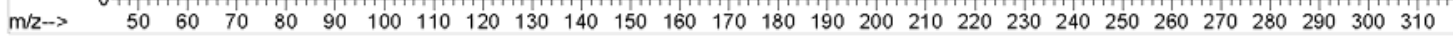

File $\quad$ :D: \DATA \TEST PT (UK) 2006\K0002919.D

Acquired : 24 Jan 2006 19:24 using AcqMethod PCI-NH3CW.M

Sample Name: 1uL of DCMP std, 145-1

Misc Info : PCI, NH3, HP-5MS

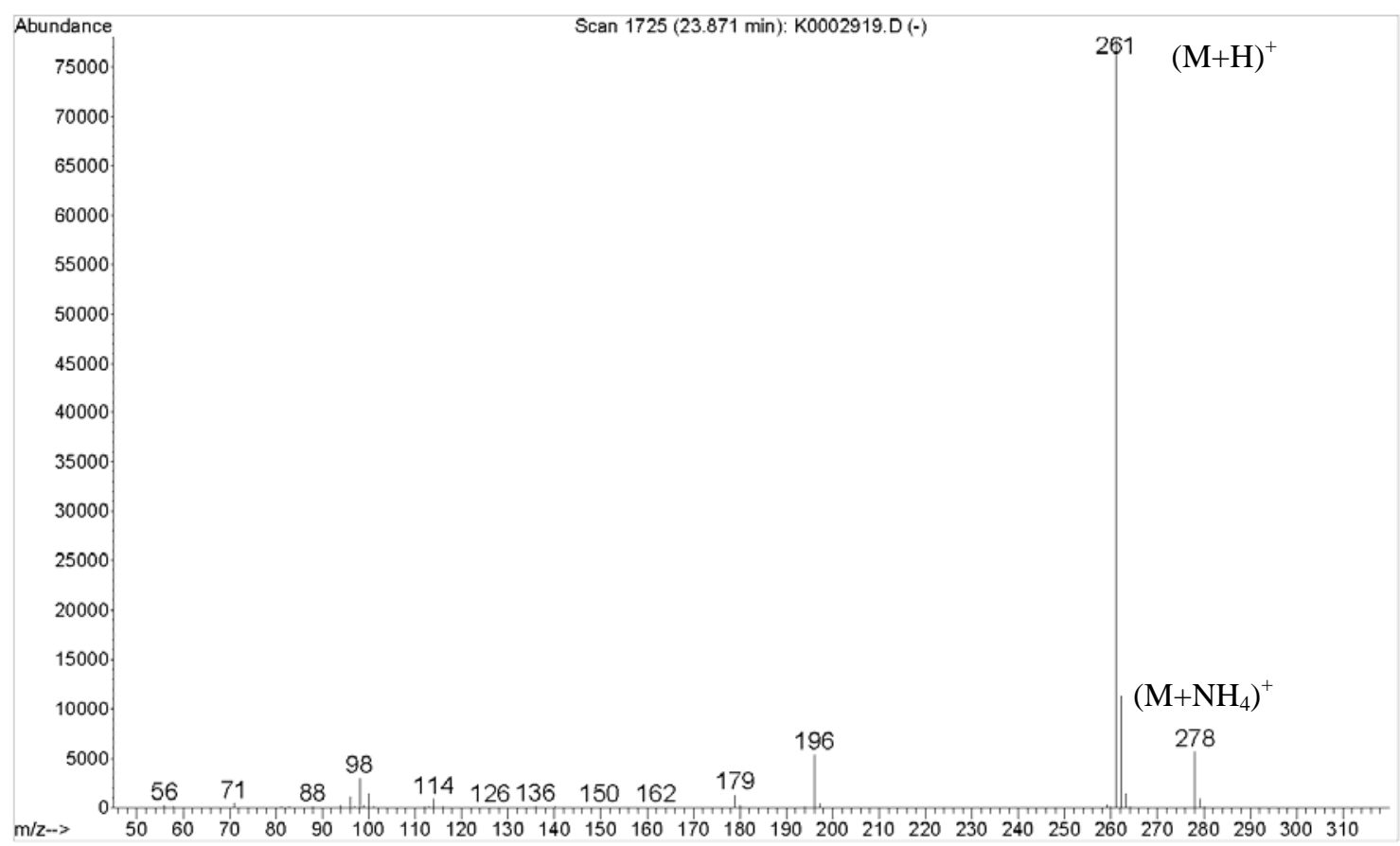

CI mass spectrum of:

Top: $\quad$ Compound 2 in Organic sample 01/05, aliquot CW-1-159-1-O1

Bottom: Authentic reference standard of Dicyclohexyl methylphosphonate corresponding to compound 2 (MW: 260) 


\section{GAS CHROMATOGRAPHY TECHNIQUE METHOD AND ANALYSIS DESCRIPTION}

Laboratory code: $\quad \underline{05}$ Sample code(s): $\underline{01 / 05}$

Chemical number:

$\underline{2}$

Aliquot codes:

Sample: $\quad$ CW-1-159-1-01

ANALYSIS METHOD

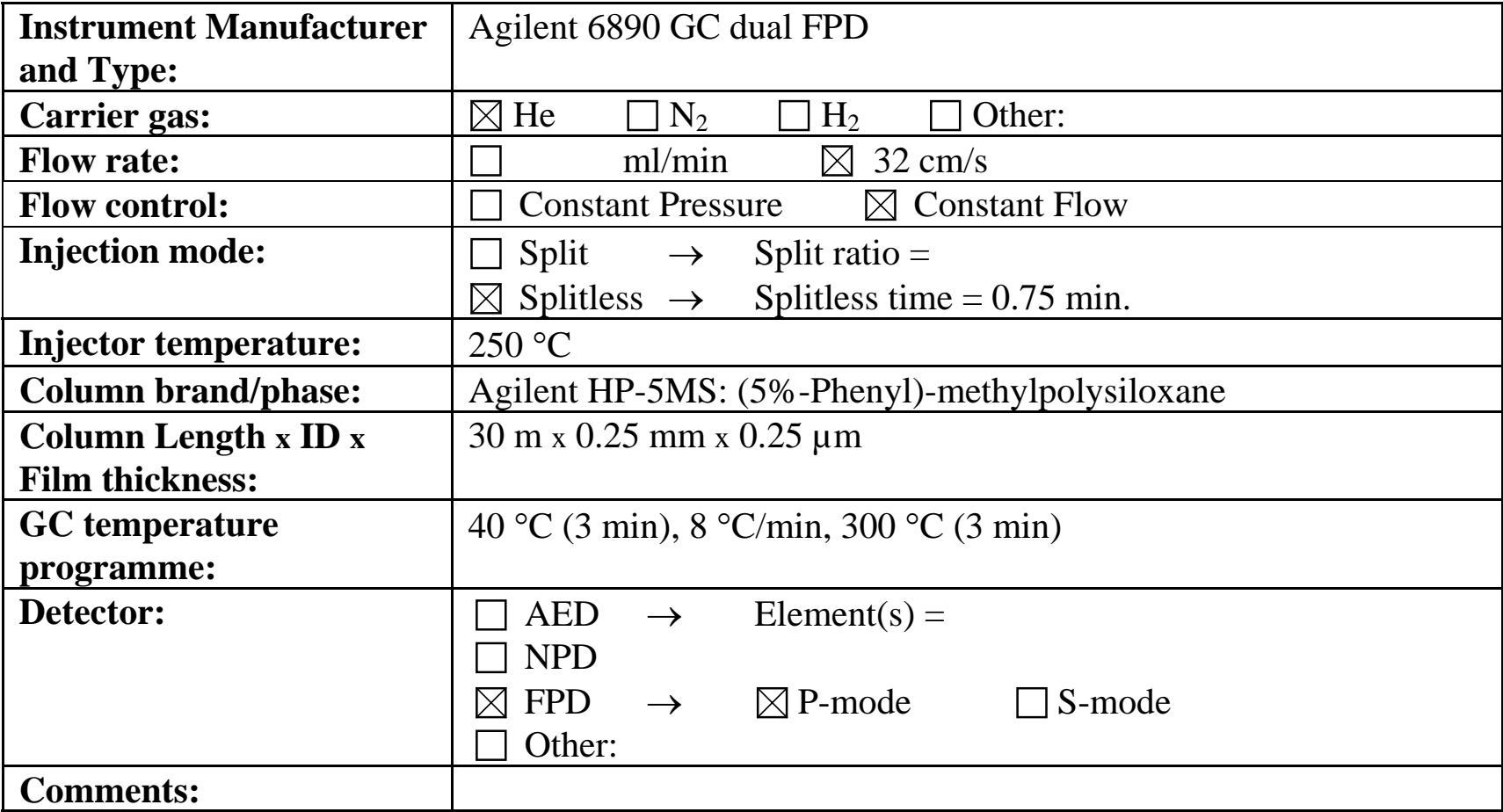

IDENTIFICATION

\begin{tabular}{|l|l|}
\hline Chemical identified as: & $\begin{array}{l}\text { Q Original Chemical } \\
\square \text { Methyl ester derivative } \\
\square \text { TBDMS (t-Butyldimethylsilyl) derivative } \\
\square \text { TMS (Trimethylsilyl) derivative } \\
\square \text { Other derivative: }\end{array}$ \\
\hline $\begin{array}{l}\text { Retention parameter used } \\
\text { for (peak) identification: }\end{array}$ & $\square$ Retention time (Rt) $\square$ Scan number \\
\hline $\begin{array}{l}\text { QCompared to reference } \\
\text { chemical: }\end{array}$ & Source of Reference: $\square$ Own Synthesis $\quad$ Commercial \\
\hline$\square$ Compared to library & $\begin{array}{l}\text { Measured RI of identified Chemical = } \\
\text { RI OCAD }=\end{array}$ \\
\hline \hline Comments & \\
\hline
\end{tabular}




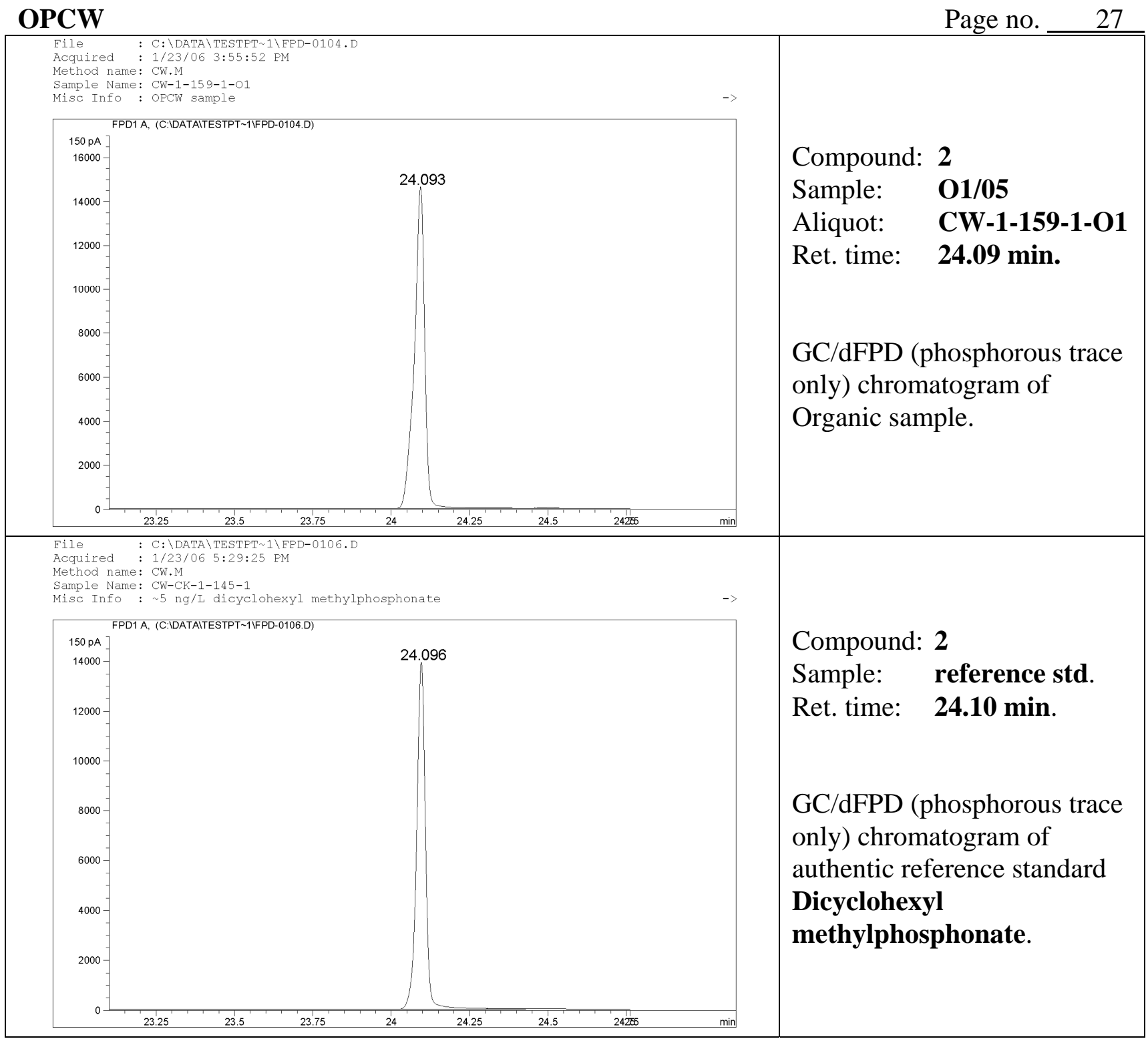




\section{GC-EI-MS TECHNIQUE METHOD AND ANALYSIS DESCRIPTION}

Laboratory code: $\quad \underline{05}$ Sample code(s): $\underline{\text { O2/05 }}$

Chemical number:

$\underline{3}$

Aliquot codes:

Sample: $\quad$ CW-1-159-2-O2

\begin{tabular}{|c|c|c|c|}
\hline $\begin{array}{l}\text { Instrument Manufacturer } \\
\text { and Type: }\end{array}$ & \multicolumn{3}{|c|}{ Agilent 6890/5973 GC/MSD } \\
\hline Carrier gas: & \multicolumn{3}{|c|}{ 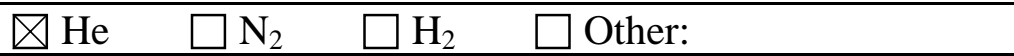 } \\
\hline Flow rate: & \multicolumn{3}{|c|}{$\mathrm{ml} / \mathrm{min} \quad \square 32 \mathrm{~cm} / \mathrm{s}$} \\
\hline Flow control: & \multicolumn{3}{|c|}{$\square$ Constant Pressure $\quad \square$ Constant Flow } \\
\hline Injection mode: & \multicolumn{3}{|c|}{$\begin{array}{llll}\square \text { Split } & \rightarrow & \text { Split ratio }= \\
\square \text { Splitless } \rightarrow & \text { Splitless time }=0.75 \mathrm{~min} .\end{array}$} \\
\hline Injector temperature: & \multicolumn{3}{|l|}{$250^{\circ} \mathrm{C}$} \\
\hline Column brand/phase: & \multicolumn{3}{|c|}{ Agilent HP-5MS: (5\%-Phenyl)-methylpolysiloxane } \\
\hline $\begin{array}{l}\text { Column Length } x \text { ID } x \\
\text { Film thickness: }\end{array}$ & \multicolumn{3}{|c|}{$30 \mathrm{~m} \times 0.25 \mathrm{~mm} \times 0.25 \mu \mathrm{m}$} \\
\hline $\begin{array}{l}\text { GC temperature } \\
\text { programme: }\end{array}$ & \multicolumn{3}{|c|}{$40^{\circ} \mathrm{C}(3 \mathrm{~min}), 8^{\circ} \mathrm{C} / \mathrm{min}, 300^{\circ} \mathrm{C}(3 \mathrm{~min})$} \\
\hline Solvent delay time: & $3 \mathrm{~min}$ & Scan range: & $30-600 \mathrm{~m} / \mathrm{z}$ \\
\hline Electron energy: & $70 \mathrm{eV}$ & Scan time: & $0.7 \mathrm{~s}$ \\
\hline Ionisation polarity: & $\begin{array}{l}\square \text { Positive } \\
\square \text { Negative }\end{array}$ & Mass resolution: & $0.7 \mathrm{u}$ \\
\hline Comments: & & & \\
\hline
\end{tabular}

IDENTIFICATION

\begin{tabular}{|c|c|}
\hline Compound identified as: & $\begin{array}{l}\text { Q Original compound } \\
\square \text { Methyl ester derivative } \\
\square \text { TBDMS (t-Butyldimethylsilyl) derivative } \\
\square \text { TMS (Trimethylsilyl) derivative } \\
\square \text { Other derivative: }\end{array}$ \\
\hline $\begin{array}{l}\text { Retention parameter used for } \\
\text { (peak) identification: }\end{array}$ & $\begin{array}{l}\bigotimes \text { Retention time (Rt) } \\
\square \text { Scan number } \\
\end{array}$ \\
\hline \Compared to reference chemical: & Source : $\square$ Own Synthesis \\
\hline$\square$ Compared to library spectrum: & $\begin{array}{lll}\text { Source : } & \square \text { OCAD (code: } & \square \text { NIST } \\
& \square \text { Wiley } \quad \square \text { Own } \quad \square \text { Other: }\end{array}$ \\
\hline $\begin{array}{l}\square \text { Not compared to reference } \\
\text { chemical or library spectrum: }\end{array}$ & $\begin{array}{l}\text { Intense ions in spectrum are explained; interpretation is } \\
\text { supported by the spectral information derived from closely } \\
\text { related chemical(s): }\end{array}$ \\
\hline
\end{tabular}




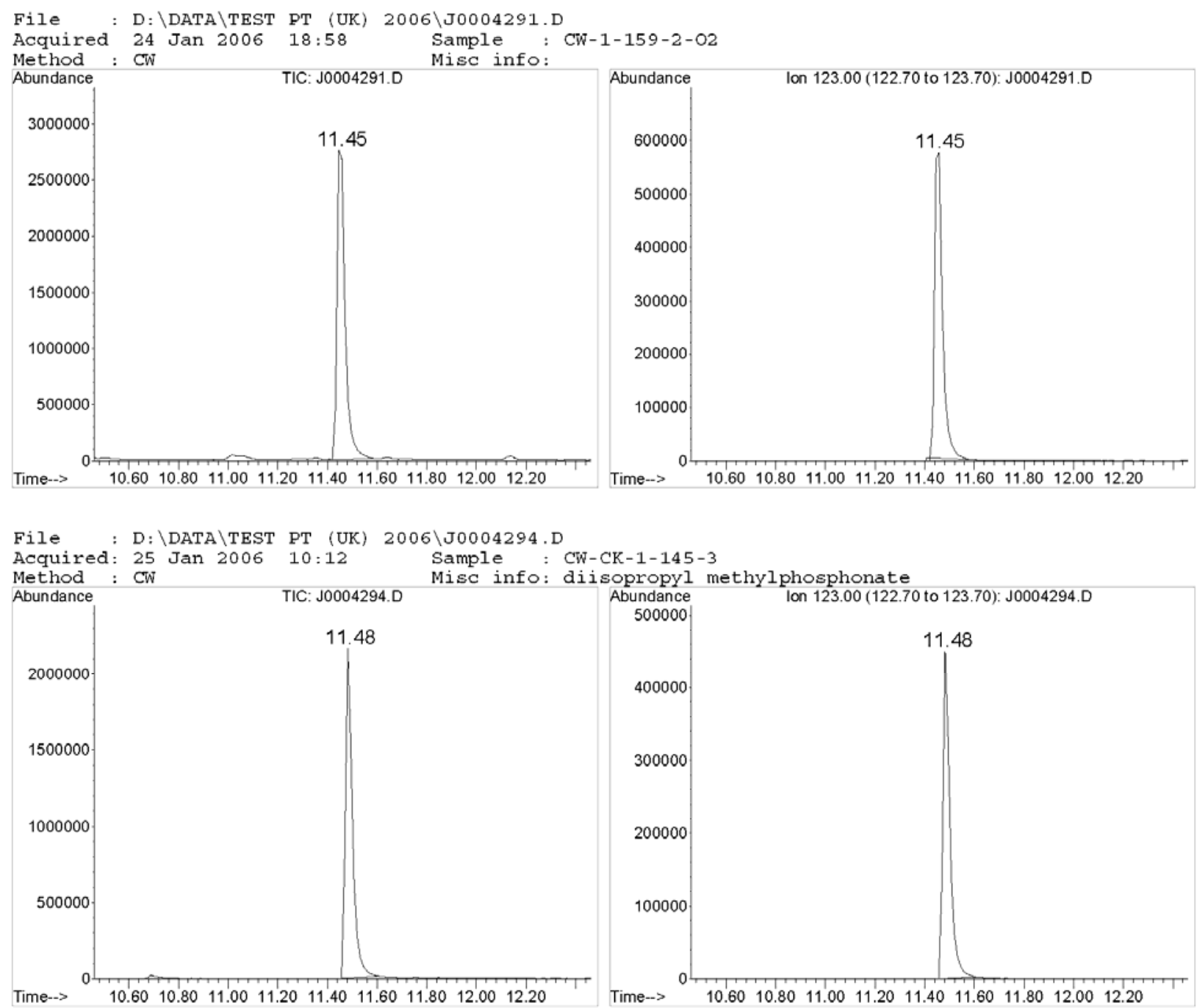

EI chromatograms supporting identification of compound 3; TIC on left; EIC (m/z 123) on right.

Top: $\quad$ Chromatograms of Organic sample, aliquot CW-1-159-2-O2 from O2/05, retention time 11.45 min.

Bottom: Chromatograms of authentic reference standard of Diisopropyl methylphosphonate, retention time 11.48 min. 
File $\quad$ :D: $\backslash$ DATA $\backslash T E S T$ PT (UK) 2006 J J0004291.D

Acquired : 24 Jan 2006 18:58 using AcqMethod CW

Sample Name: CW-1-159-2-O2

Misc Info :

Abundance Scan $569(11.445 \mathrm{~min}):$ J0004291.D (-)

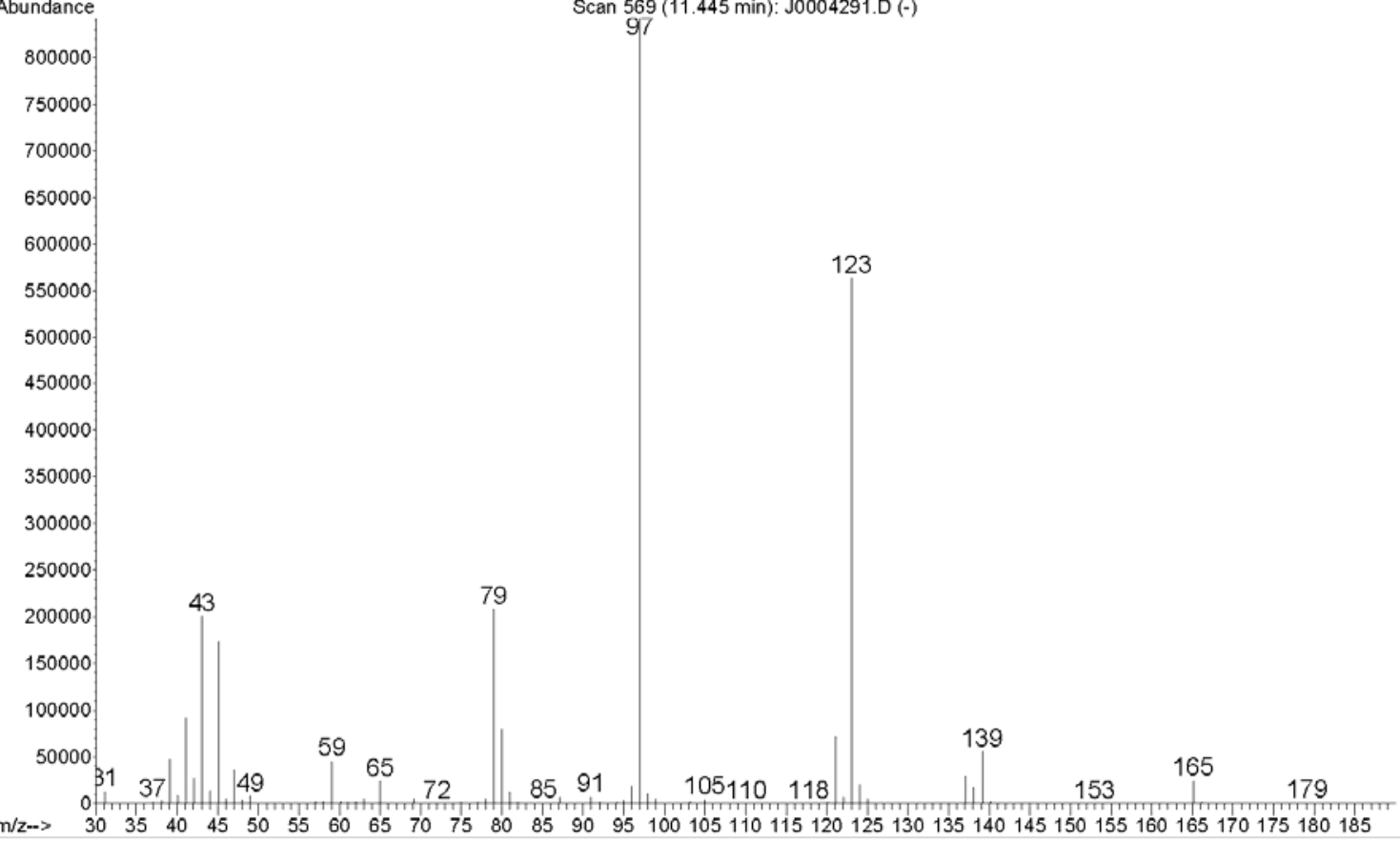

File $\quad$ :D: \DATA \TEST PT (UK) 2006\J0004294.D

Acquired : 25 Jan 2006 10:12 using AcqMethod CW

Sample Name: CW-CK-1-145-3

Misc Info : diisopropyl methylphosphonate

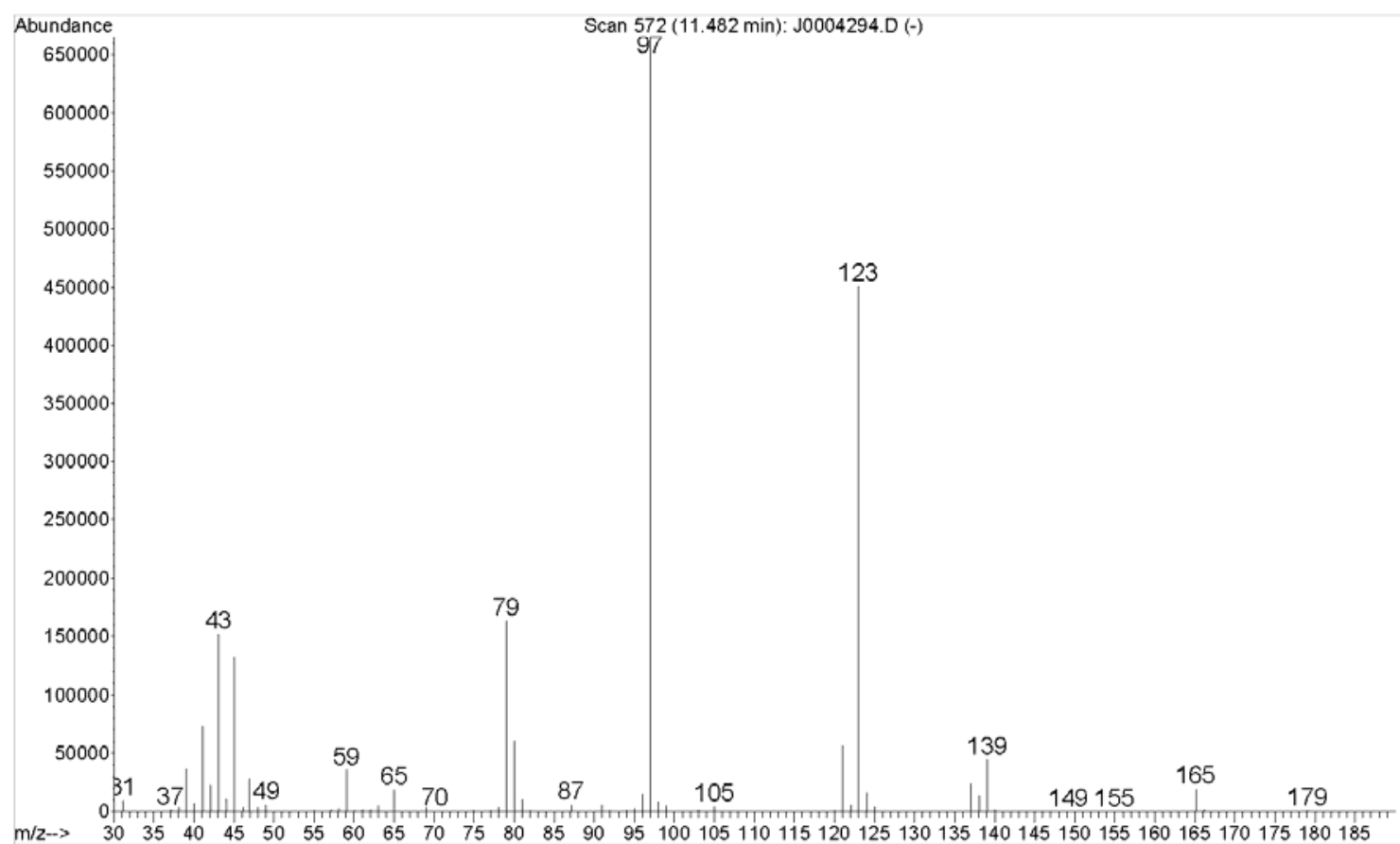

EI mass spectra of:

Top: $\quad$ Compound 3 in Organic sample O2/05, aliquot CW-1-159-2-O2

Bottom: Authentic reference standard of Diisopropyl methylphosphonate corresponding to compound 3 (MW: 180) 


\section{GC-CI-MS TECHNIQUE METHOD AND ANALYSIS DESCRIPTION}

Laboratory code: $\underline{05}$ Sample code(s): $\underline{\text { O2/05 }}$

Chemical number:

$\underline{3}$

Aliquot codes:

Sample: $\quad$ CW-1-159-2-O2

ANALYSIS METHOD

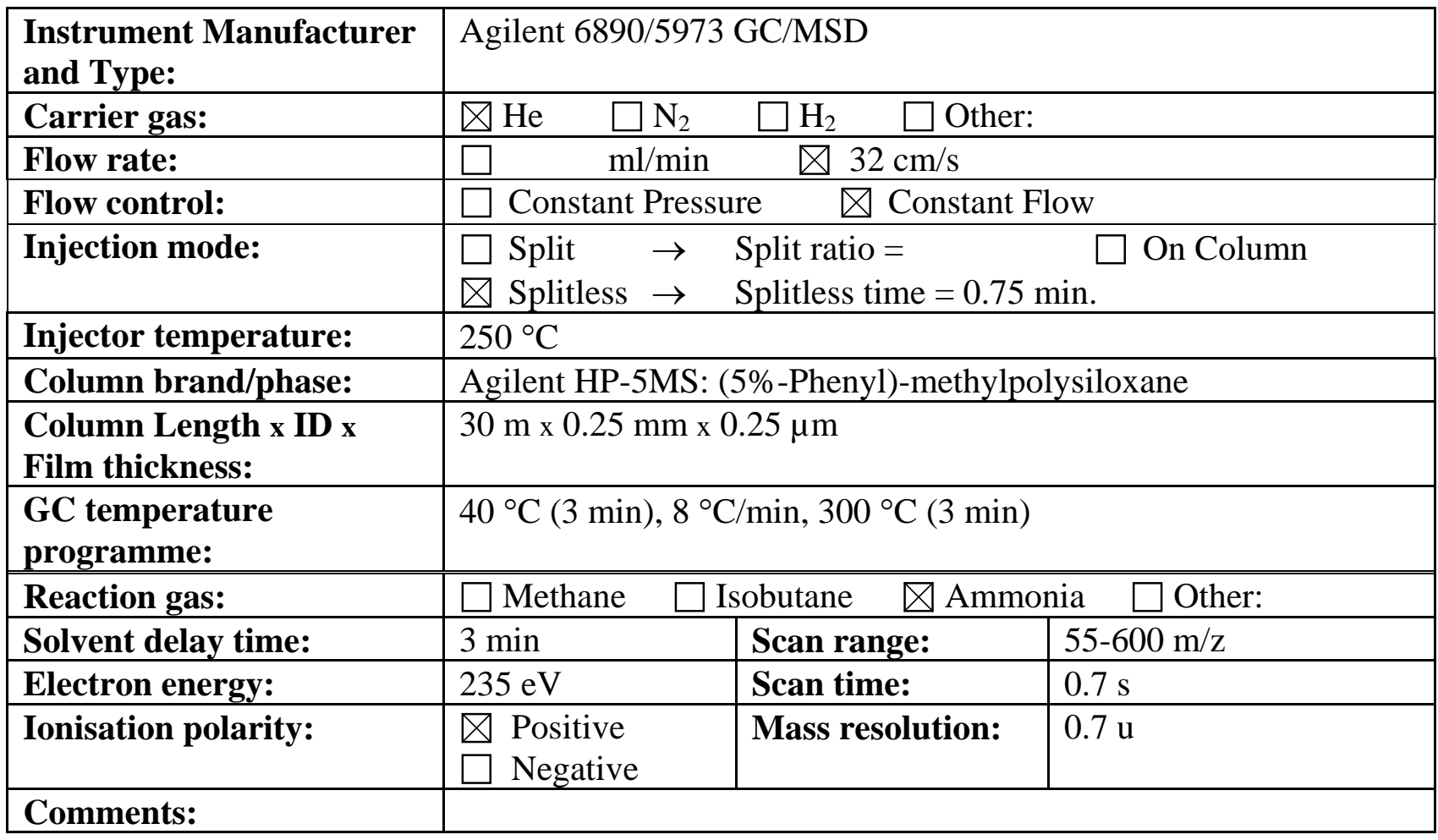

IDENTIFICATION

\begin{tabular}{|l|l|}
\hline Compound identified as: & $\square$ Original compound \\
& $\square$ Methyl ester derivative \\
& $\square$ TBDMS (t-Butyldimethylsilyl) derivative \\
& $\square$ TMS (Trimethylsilyl) derivative \\
& $\square$ Other derivative: \\
\hline $\begin{array}{l}\text { Retention parameter used for } \\
\text { (peak) identification: }\end{array}$ & $\square$ Retention time (Rt) \\
\hline \hline$\square$ Compared to reference chemical: & Source : $\square$ Own Synthesis $\square$ Commercial \\
\hline $\begin{array}{l}\text { Not compared to reference } \\
\text { chemical or library spectrum: }\end{array}$ & Intense ions in spectrum are explained \\
\hline Comments: & $\square$ RT GC/MS-EI \\
\hline
\end{tabular}




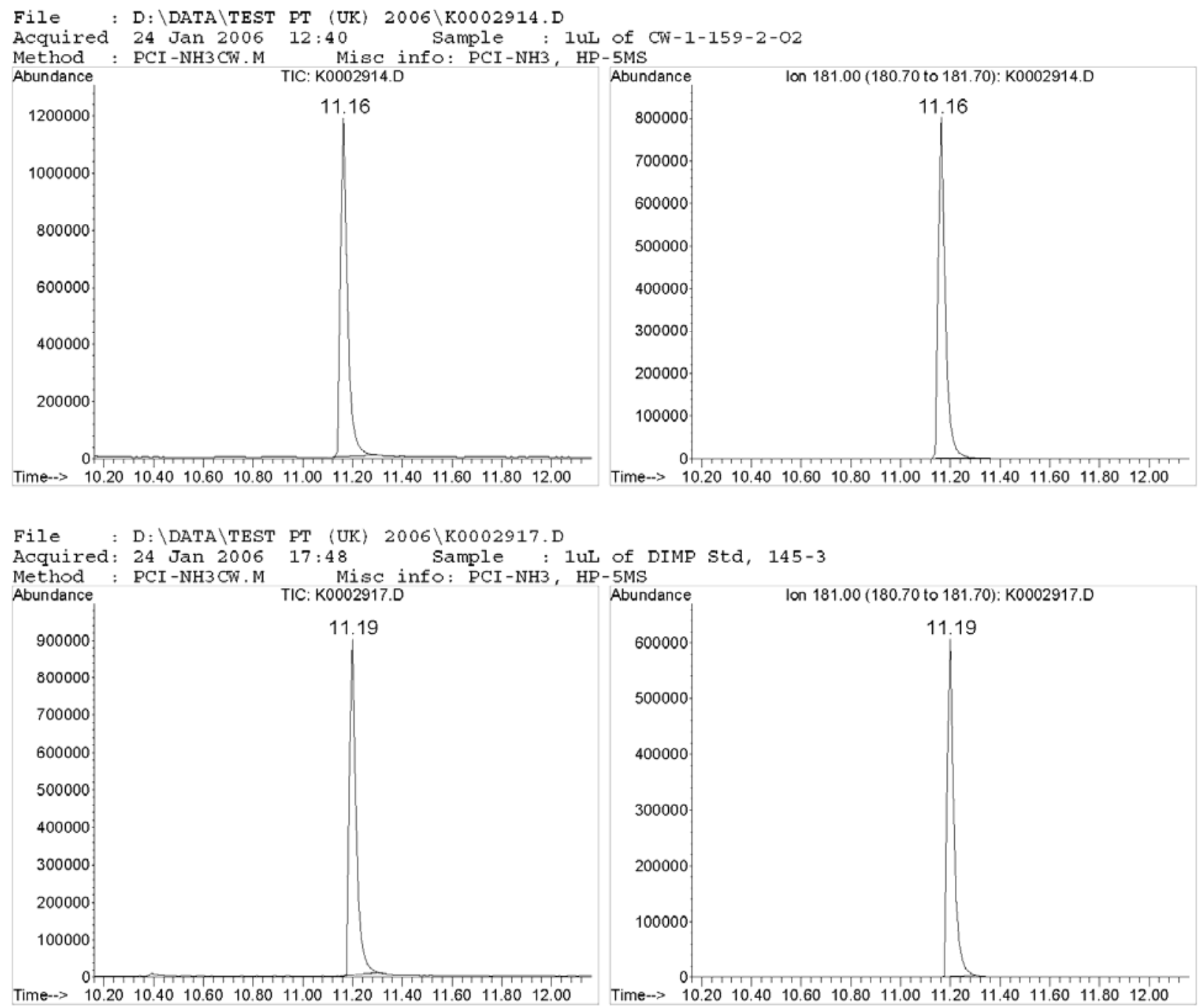

CI chromatograms supporting identification of compound 3; TIC on left; EIC (m/z 181) on right.

Top: $\quad$ Chromatograms of Organic sample, aliquot CW-1-159-2-O2 from O2/05, retention time 11.16 min.

Bottom: Chromatograms of authentic reference standard of Diisopropyl methylphosphonate, retention time 11.19 min. 


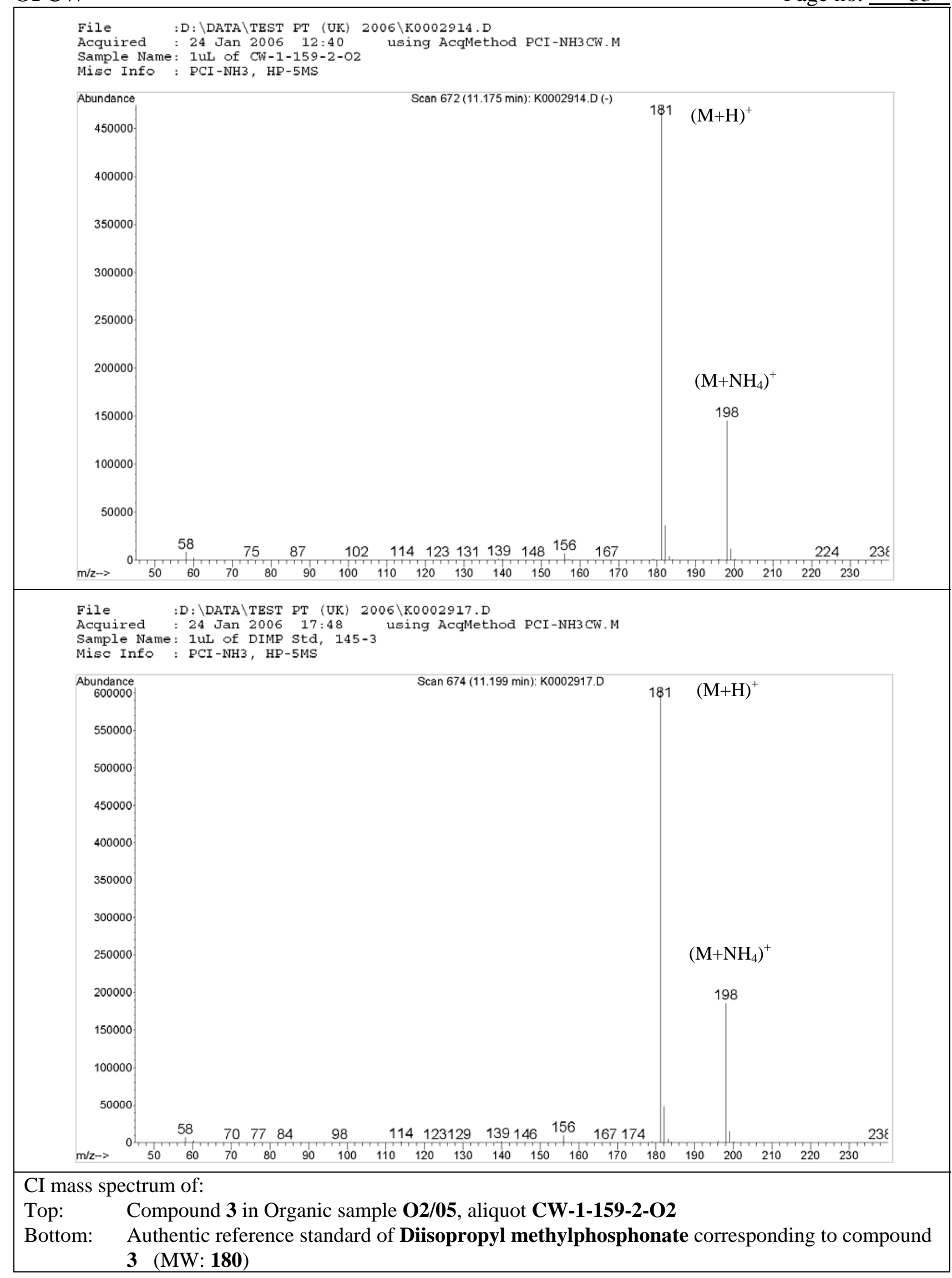




\section{GAS CHROMATOGRAPHY TECHNIQUE METHOD AND ANALYSIS DESCRIPTION}

Laboratory code: $\quad \underline{05}$ Sample code(s): $\underline{\text { O2/05 }}$

Chemical number:

$\underline{3}$

\section{Aliquot codes:}

Sample: $\quad$ CW-1-159-2-O2

\begin{tabular}{|c|c|}
\hline $\begin{array}{l}\text { Instrument Manufacturer } \\
\text { and Type: }\end{array}$ & Agilent 6890 GC dual FPD \\
\hline Carrier gas: & $\begin{array}{lll}\triangle \mathrm{He} & \square \mathrm{N}_{2}\end{array} \mathrm{H}_{2} \quad \square$ Other: \\
\hline Flow rate: & $\square \quad \mathrm{ml} / \mathrm{min} \quad \triangle 32 \mathrm{~cm} / \mathrm{s}$ \\
\hline Flow control: & $\square$ Constant Pressure \\
\hline Injection mode: & $\begin{array}{lll}\square \text { Split } & \rightarrow & \text { Split ratio }= \\
\square \text { Splitless } & \rightarrow & \text { Splitless time }=0.75 \mathrm{~min}\end{array}$ \\
\hline Injector temperature: & $250^{\circ} \mathrm{C}$ \\
\hline Column brand/phase: & Agilent HP-5MS: (5\%-Phenyl)-methylpolysiloxane \\
\hline $\begin{array}{l}\text { Column Length } \mathrm{x} \text { ID } \mathrm{x} \\
\text { Film thickness: }\end{array}$ & $30 \mathrm{~m} \times 0.25 \mathrm{~mm} \times 0.25 \mu \mathrm{m}$ \\
\hline $\begin{array}{l}\text { GC temperature } \\
\text { programme: }\end{array}$ & $40^{\circ} \mathrm{C}(3 \mathrm{~min}), 8^{\circ} \mathrm{C} / \mathrm{min}, 300{ }^{\circ} \mathrm{C}(3 \mathrm{~min})$ \\
\hline Detector: & $\begin{array}{l}\square \text { AED } \quad \rightarrow \quad \text { Element(s)= } \\
\square \text { NPD } \\
\square \text { FPD } \rightarrow \quad \text { \P-mode } \quad \square \text { S-mode } \\
\square \text { Other: }\end{array}$ \\
\hline Comments: & \\
\hline
\end{tabular}

IDENTIFICATION

\begin{tabular}{|l|l|}
\hline Chemical identified as: & $\begin{array}{l}\text { Q Original Chemical } \\
\square \text { Methyl ester derivative } \\
\square \text { TBDMS (t-Butyldimethylsilyl) derivative } \\
\square \text { TMS (Trimethylsilyl) derivative } \\
\square \text { Other derivative: }\end{array}$ \\
\hline $\begin{array}{l}\text { Retention parameter used } \\
\text { for (peak) identification: }\end{array}$ & $\square$ Retention time (Rt) $\square$ Scan number \\
\hline $\begin{array}{l}\text { Q Compared to reference } \\
\text { chemical: }\end{array}$ & Source of Reference: $\square$ Own Synthesis $\quad$ Commercial \\
\hline $\begin{array}{l}\square \text { Compared to library } \\
\text { RI: }\end{array}$ & $\begin{array}{l}\text { Measured RI of identified Chemical }= \\
\text { RI OCAD }=\end{array}$ \\
\hline \hline Comments & \\
\hline
\end{tabular}




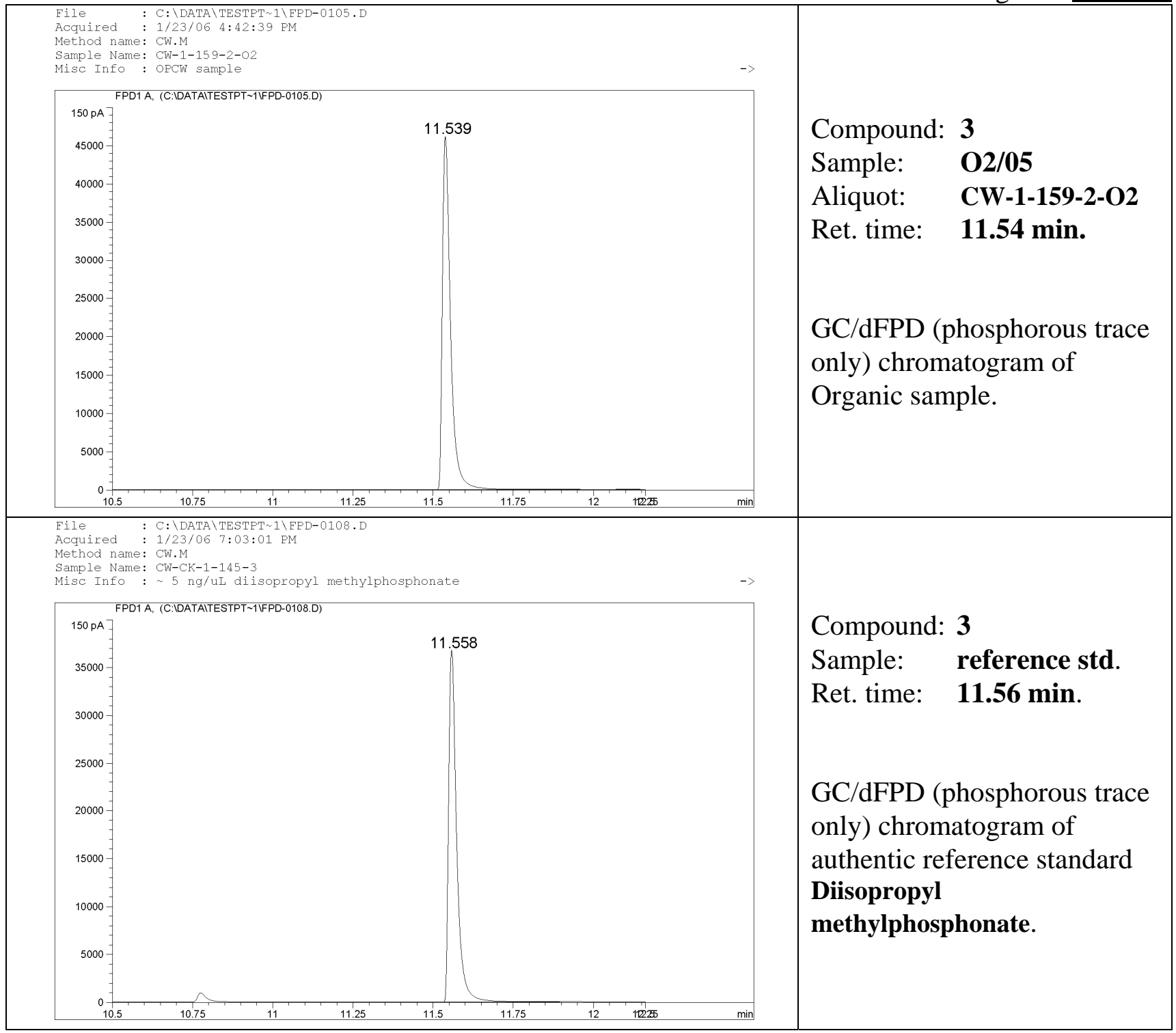




\section{GC-EI-MS TECHNIQUE METHOD AND ANALYSIS DESCRIPTION}

Laboratory code: $\quad \underline{05}$ Sample code(s): $\quad \underline{\text { O2/05 }}$

Chemical number:

$\underline{4}$

Aliquot codes:

Sample: $\quad$ CW-1-159-2-O2

ANALYSIS METHOD

\begin{tabular}{|c|c|c|c|}
\hline Instrument Manufacturer & \multicolumn{3}{|c|}{ Agilent 6890/5973 GC/MSD } \\
\hline Carrier gas: & $\square \mathrm{N}_{2}$ & \multicolumn{2}{|l|}{$\square \mathrm{H}_{2} \quad \square$ Other: } \\
\hline Flow rate: & $\mathrm{ml} / \mathrm{min}$ & \multicolumn{2}{|l|}{$\triangle 32 \mathrm{~cm} / \mathrm{s}$} \\
\hline Flow control: & $\square$ Constant Press & \multicolumn{2}{|c|}{$\triangle$ Constant Flow } \\
\hline Injection mode: & $\begin{array}{ll}\square \text { Split } & \rightarrow \\
\triangle \text { Splitless } & \rightarrow\end{array}$ & \multicolumn{2}{|c|}{$\begin{array}{l}\text { Split ratio }=\quad \square \text { On Column } \\
\text { Splitless time }=0.75 \mathrm{~min} .\end{array}$} \\
\hline Injector temperature: & \multicolumn{3}{|l|}{$250^{\circ} \mathrm{C}$} \\
\hline Column brand/phase: & \multicolumn{3}{|c|}{ Agilent HP-5MS: (5\%-Phenyl)-methylpolysiloxane } \\
\hline $\begin{array}{l}\text { Column Length } \mathrm{x} \text { ID } \mathrm{x} \\
\text { Film thickness: }\end{array}$ & \multicolumn{3}{|c|}{$30 \mathrm{~m} \times 0.25 \mathrm{~mm} \times 0.25 \mu \mathrm{m}$} \\
\hline $\begin{array}{l}\text { GC temperature } \\
\text { programme: }\end{array}$ & \multicolumn{3}{|c|}{$40^{\circ} \mathrm{C}(3 \mathrm{~min}), 8{ }^{\circ} \mathrm{C} / \mathrm{min}, 300^{\circ} \mathrm{C}(3 \mathrm{~min})$} \\
\hline Solvent delay time: & $3 \mathrm{~min}$ & Scan range: & $30-600 \mathrm{~m} / \mathrm{z}$ \\
\hline Electron energy: & $70 \mathrm{eV}$ & Scan time: & $0.7 \mathrm{~s}$ \\
\hline Ionisation polarity: & $\begin{array}{l}\text { Positive } \\
\square \text { Negative }\end{array}$ & Mass resolution: & $0.7 \mathrm{u}$ \\
\hline
\end{tabular}

IDENTIFICATION

\begin{tabular}{|c|c|}
\hline Compound identified as: & $\begin{array}{l}\bigotimes \text { Original compound } \\
\square \text { Methyl ester derivative } \\
\square \text { TBDMS (t-Butyldimethylsilyl) derivative } \\
\square \text { TMS (Trimethylsilyl) derivative } \\
\square \text { Other derivative: }\end{array}$ \\
\hline $\begin{array}{l}\text { Retention parameter used for } \\
\text { (peak) identification: }\end{array}$ & $\begin{array}{l}\square \text { Retention time (Rt) } \\
\square \text { Scan number }\end{array}$ \\
\hline$\triangle$ Compared to reference chemical: & Source : $\searrow$ Own Synthesis \\
\hline$\square$ Compared to library spectrum: & $\begin{array}{lll}\text { Source : } & \square \text { OCAD (code: } & \square \text { ) } \\
& \square \text { WisT } \\
& \square \text { Wiley } \quad \square \text { Own } & \square \text { Other: }\end{array}$ \\
\hline $\begin{array}{l}\square \text { Not compared to reference } \\
\text { chemical or library spectrum: }\end{array}$ & $\begin{array}{l}\text { Intense ions in spectrum are explained; interpretation is } \\
\text { supported by the spectral information derived from closely } \\
\text { related chemical(s): }\end{array}$ \\
\hline
\end{tabular}




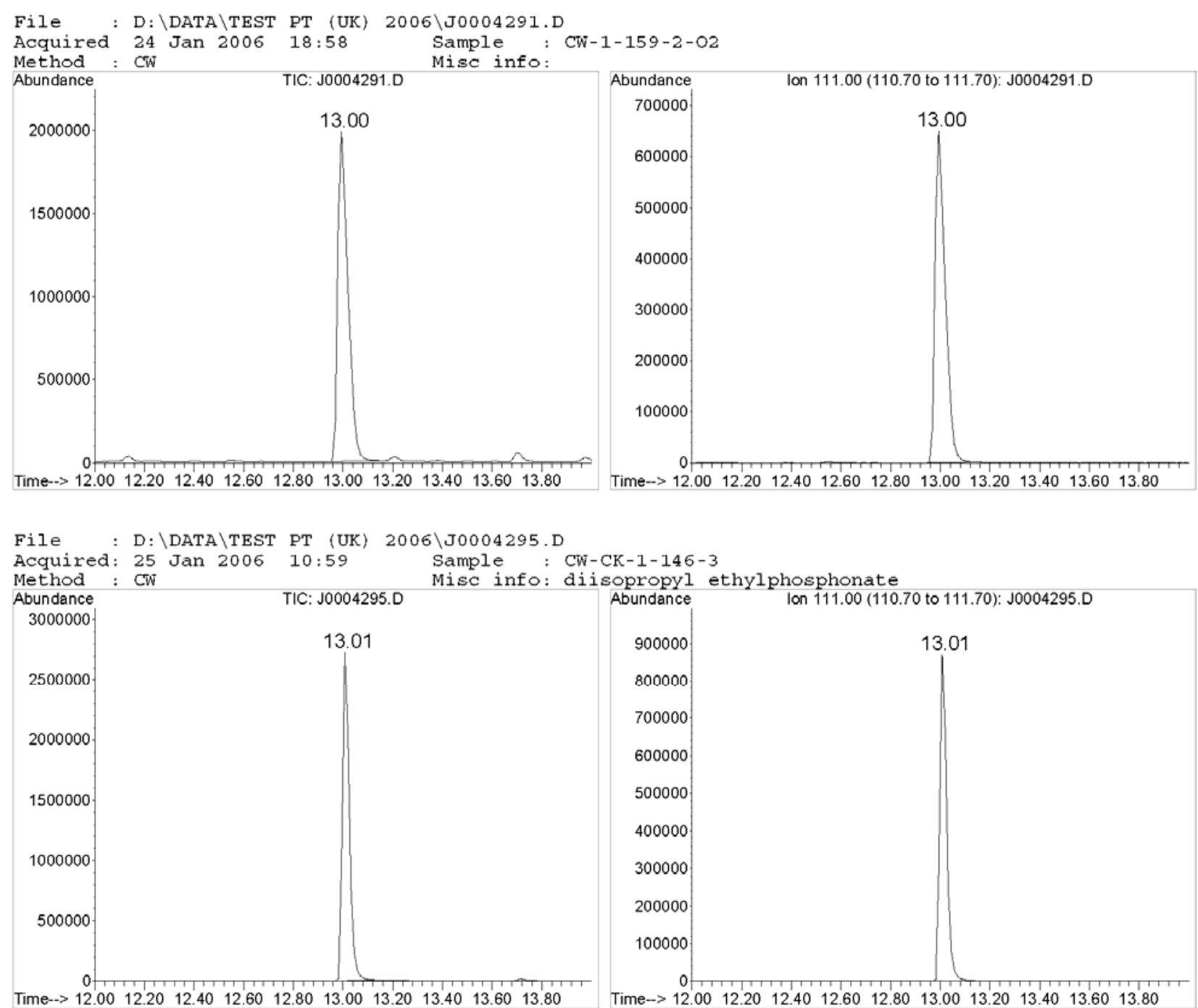

EI chromatograms supporting identification of compound 4; TIC on left; EIC (m/z 111) on right.

Top: $\quad$ Chromatograms of Organic sample, aliquot CW-1-159-2-O2 from O2/05, retention time 13.00 min.

Bottom: Chromatograms of authentic reference standard of Diisopropyl ethylphosphonate, retention time 13.01 min. 


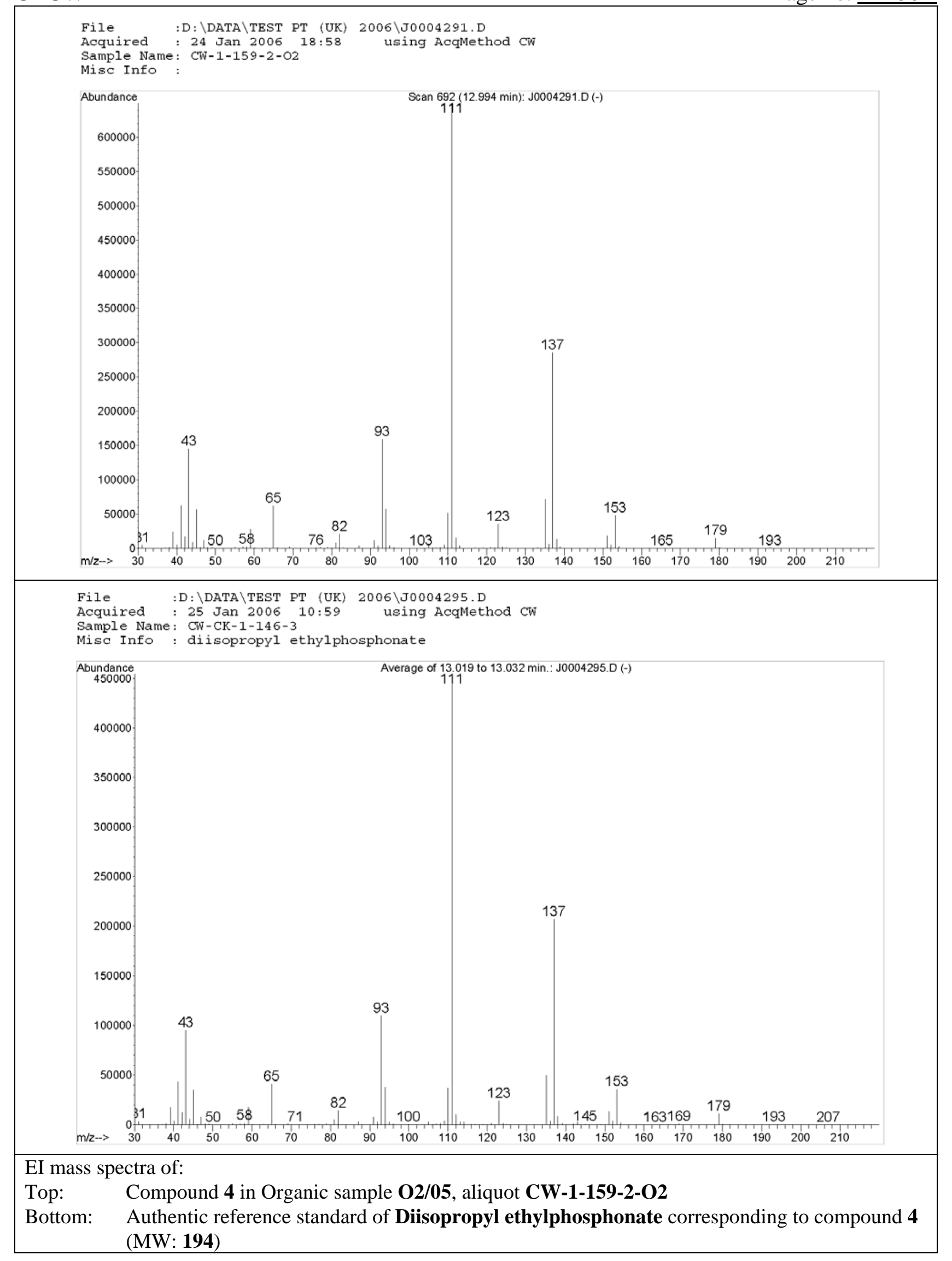




\section{GC-CI-MS TECHNIQUE METHOD AND ANALYSIS DESCRIPTION}

Laboratory code: $\underline{05}$ Sample code(s): $\underline{\text { O2/05 }}$

Chemical number:

$\underline{4}$

Aliquot codes:

Sample: $\quad$ CW-1-159-2-O2

ANALYSIS METHOD

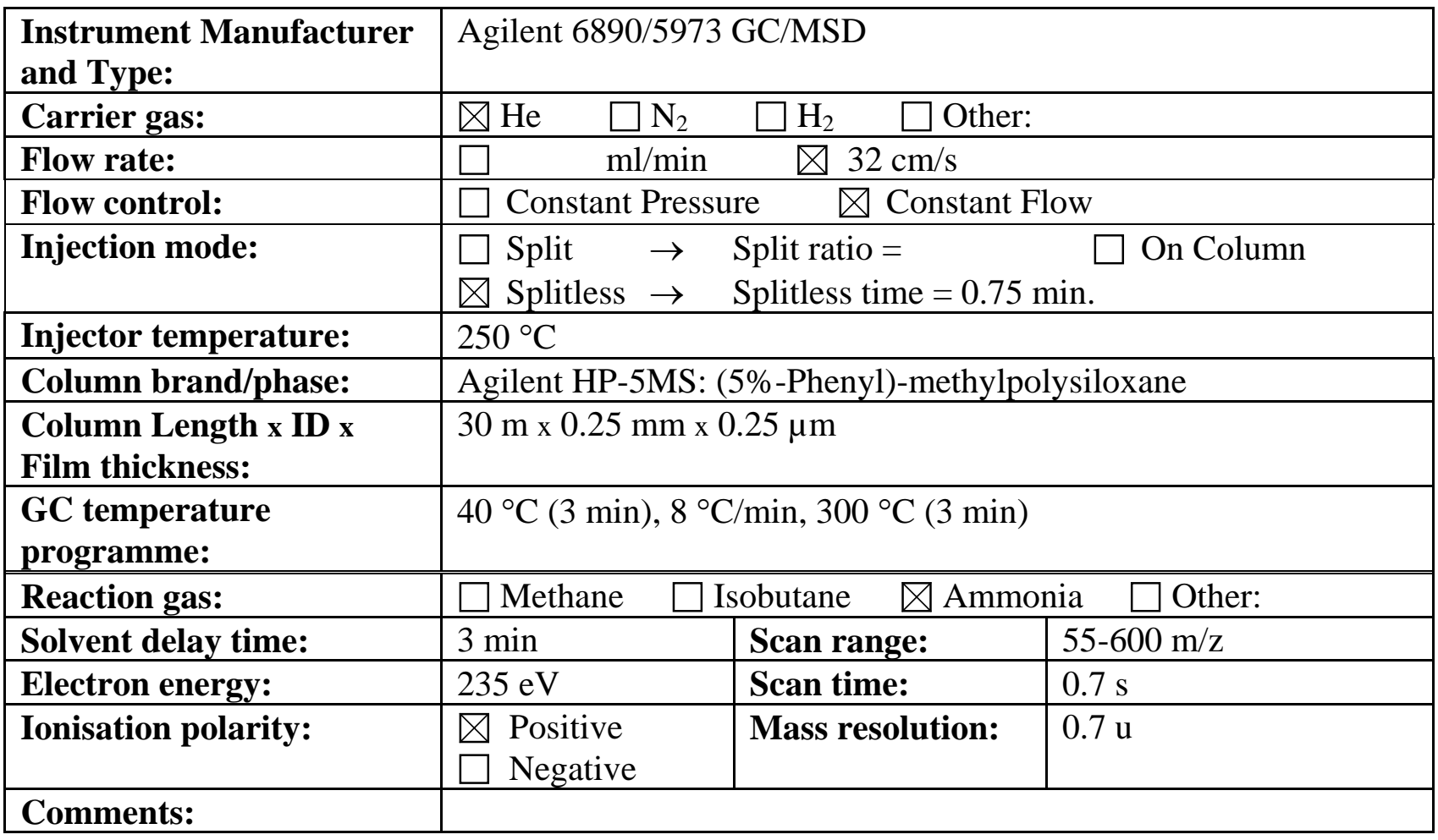

IDENTIFICATION

\begin{tabular}{|l|l|}
\hline Compound identified as: & Q Original compound \\
& $\square$ Methyl ester derivative \\
& $\square$ TBDMS (t-Butyldimethylsilyl) derivative \\
& $\square$ TMS (Trimethylsilyl) derivative \\
& $\square$ Other derivative: \\
\hline $\begin{array}{l}\text { Retention parameter used for } \\
\text { (peak) identification: }\end{array}$ & $\square$ Retention time (Rt) \\
\hline \hline$\square$ Compared to reference chemical: & Source : S Own Synthesis $\square$ Commercial \\
\hline $\begin{array}{l}\text { Not compared to reference } \\
\text { chemical or library spectrum: }\end{array}$ & Intense ions in spectrum are explained \\
\hline Comments: & $\square$ RT GC/MS-EI \\
\hline
\end{tabular}




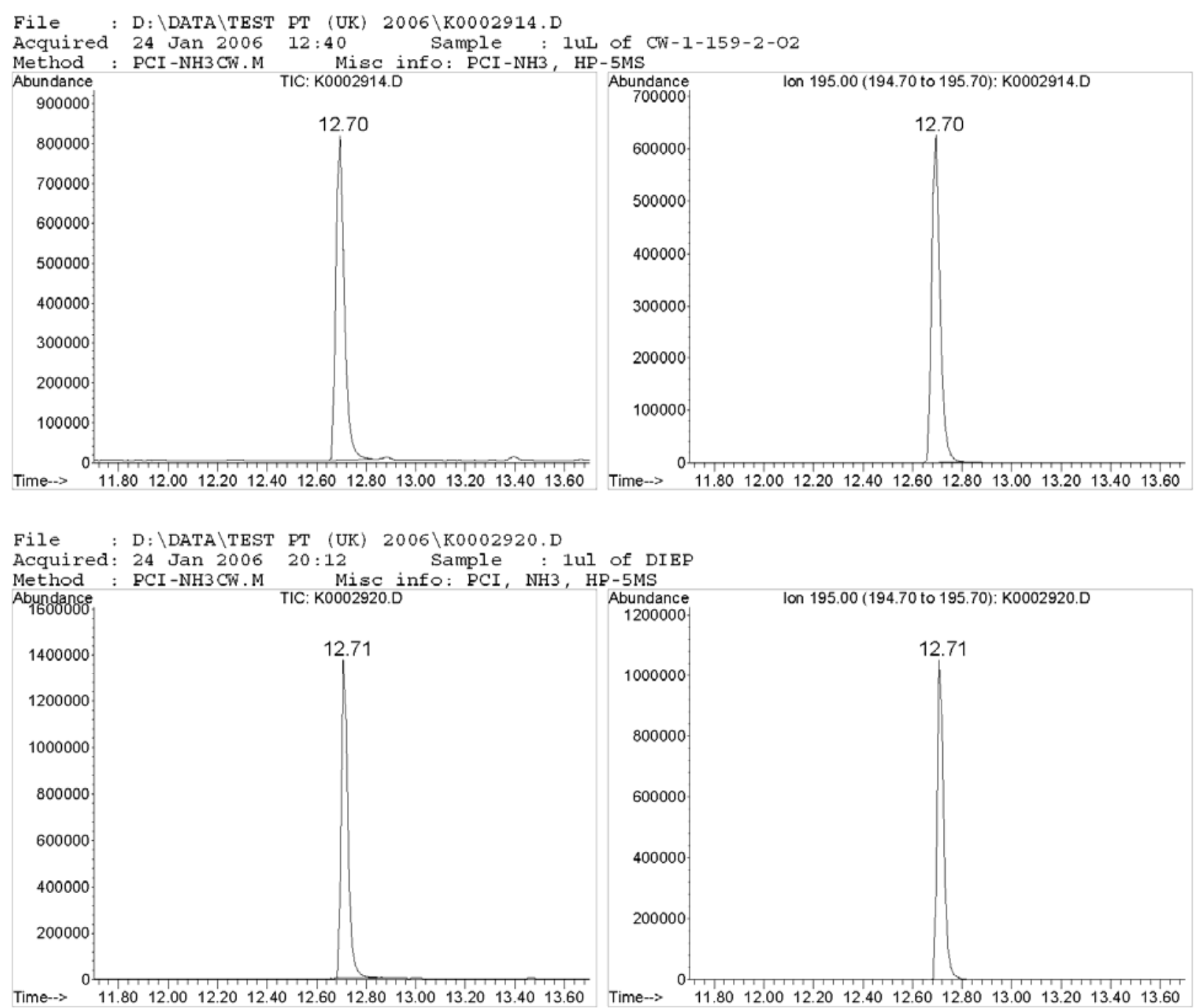

CI chromatograms supporting identification of compound 4; TIC on left; EIC (m/z 195) on right.

Top: $\quad$ Chromatograms of Organic sample, aliquot CW-1-159-2-O2 from O2/05, retention time 12.70 min.

Bottom: Chromatograms of authentic reference standard of Diisopropyl ethylphosphonate, retention time 12.71 min. 


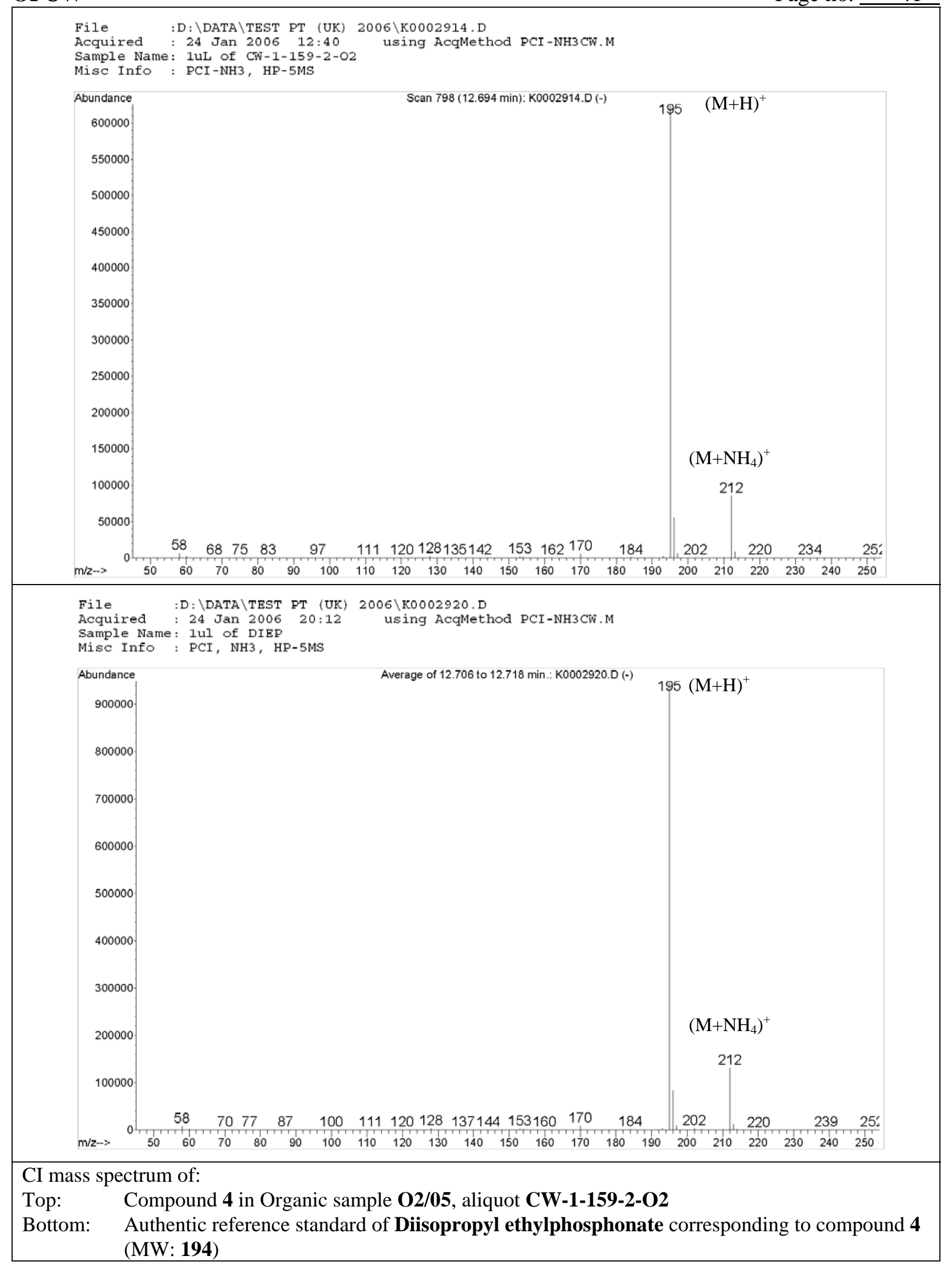




\section{GAS CHROMATOGRAPHY TECHNIQUE METHOD AND ANALYSIS DESCRIPTION}

Laboratory code: $\quad \underline{05}$ Sample code(s): $\quad \underline{02 / 05}$

Chemical number:

$\underline{4}$

Aliquot codes:

Sample: $\quad$ CW-1-159-2-O2

ANALYSIS METHOD

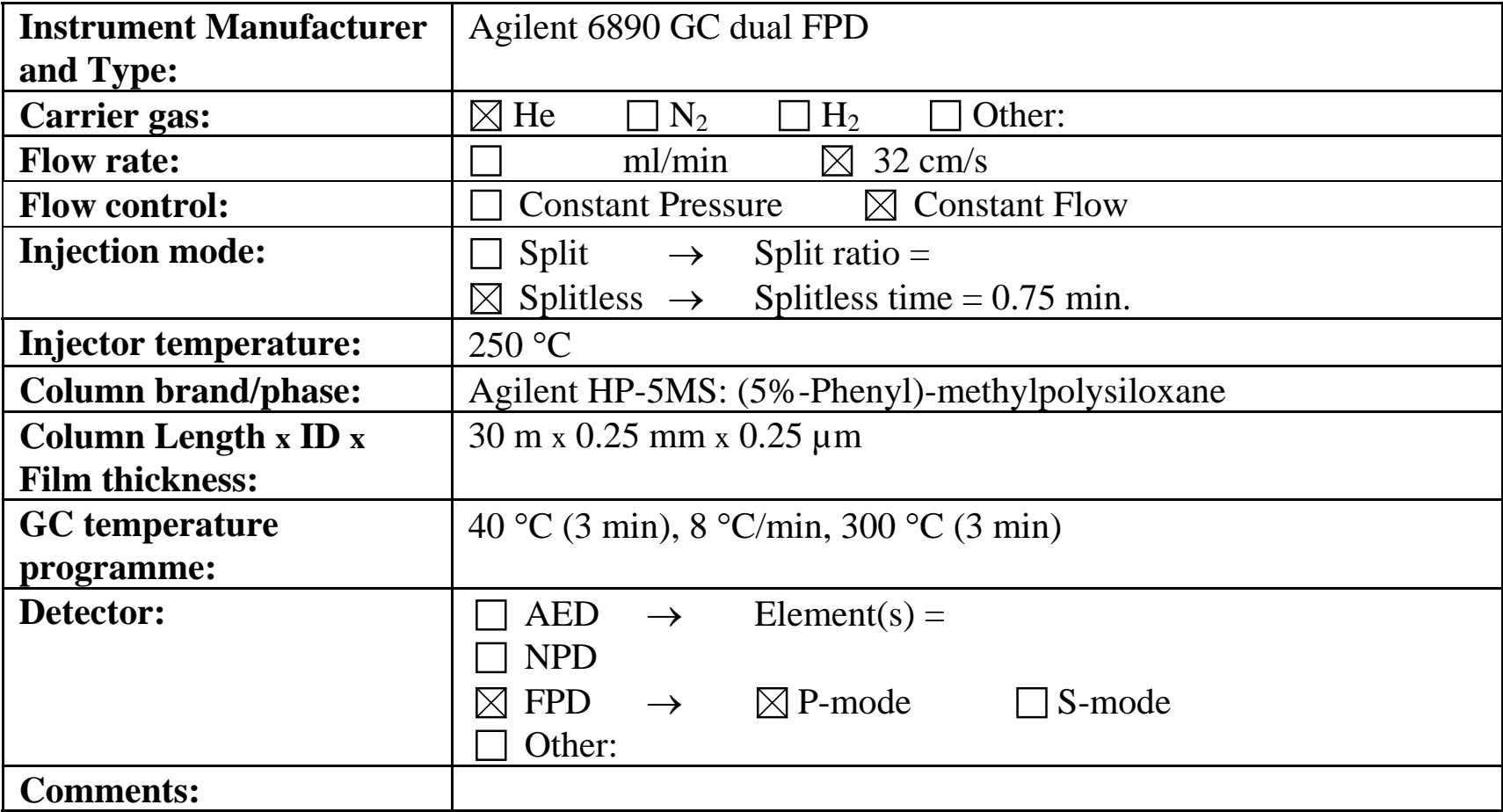

IDENTIFICATION

\begin{tabular}{|l|l|}
\hline Chemical identified as: & $\begin{array}{l}\text { Q Original Chemical } \\
\square \text { Methyl ester derivative } \\
\square \text { TBDMS (t-Butyldimethylsilyl) derivative } \\
\square \text { TMS (Trimethylsilyl) derivative } \\
\square \text { Other derivative: }\end{array}$ \\
\hline $\begin{array}{l}\text { Retention parameter used } \\
\text { for (peak) identification: }\end{array}$ & $\square$ Retention time (Rt) $\square$ Scan number \\
\hline $\begin{array}{l}\text { Q Compared to reference } \\
\text { chemical: }\end{array}$ & Source of Reference: $\quad$ Own Synthesis $\square$ Commercial \\
\hline $\begin{array}{l}\square \text { Compared to library } \\
\text { RI: }\end{array}$ & $\begin{array}{l}\text { Measured RI of identified Chemical }= \\
\text { RI OCAD }=\end{array}$ \\
\hline \hline Comments & \\
\hline
\end{tabular}




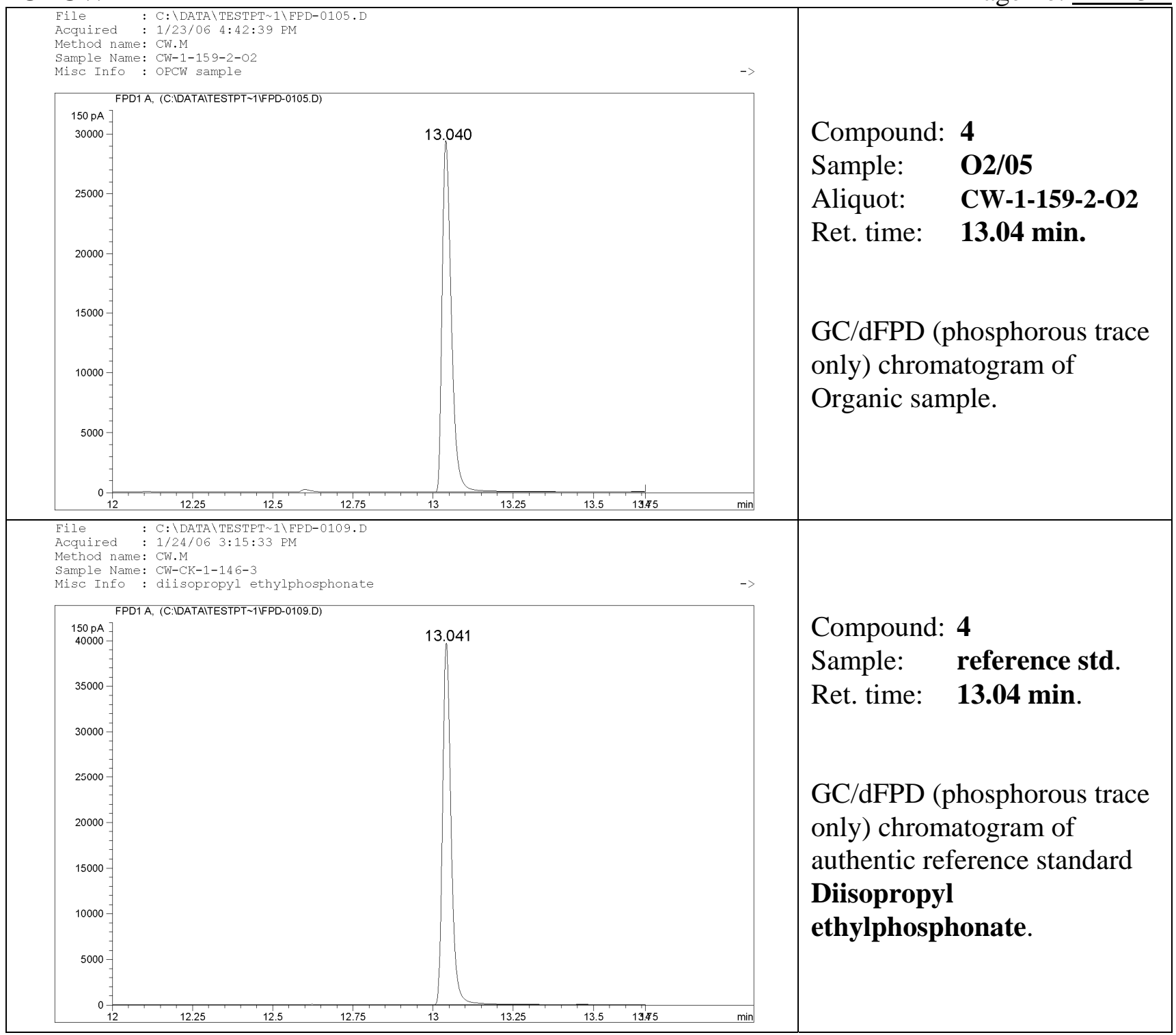




\section{COMMENTS}

\section{General}

No relevant CWC schedule compounds were identified in sample O3/05

\section{Sample preparation}

None

\section{Analysis}

No additional comments

\section{Report}

The field length in the Chemstation software often did not allow for the full chemical name and may be truncated or abbreviated.

The GC Chemstation report software often truncated the time axis at approximately $80 \%$ of full scale; in no case was data of significance not displayed correctly. 